\title{
Drosophila Acyl-CoA Synthetase Long-Chain Family Member 4 Regulates Axonal Transport of Synaptic Vesicles and Is Required for Synaptic Development and Transmission
}

\author{
Zhihua Liu, Yan Huang, Yi Zhang, Di Chen, and Yong Q. Zhang \\ Key Laboratory for Molecular and Developmental Biology, Institute of Genetics and Developmental Biology, Chinese Academy of Sciences, Beijing 100101, \\ China
}

Acyl-CoA synthetase long-chain family member 4 (ACSL4) converts long-chain fatty acids to acyl-CoAs that are indispensable for lipid metabolism and cell signaling. Mutations in ACSL4 cause nonsyndromic X-linked mental retardation. We previously demonstrated that Drosophila dAcsl is functionally homologous to human ACSL4, and is required for axonal targeting in the brain. Here, we report that Drosophila dAcsl mutants exhibited distally biased axonal aggregates that were immunopositive for the synaptic-vesicle proteins synaptotagmin (Syt) and cysteine-string protein, the late endosome/lysosome marker lysosome-associated membrane protein 1, the autophagosomal marker Atg8, and the multivesicular body marker Hrs (hepatocyte growth factor-regulated tyrosine kinase substrate). In contrast, the axonal distribution of mitochondria and the cell adhesion molecule Fas II (fasciclin II) was normal. Electron microscopy revealed accumulation of prelysomes and multivesicle bodies. These aggregates appear as retrograde instead of anterograde cargos. Live imaging analysis revealed that $d A c s l$ mutations increased the velocity of anterograde transport but reduced the flux, velocity, and processivity of retrograde transport of Syt-enhanced green fluorescent protein-labeled vesicles. Immunohistochemical and electrophysiological analyses showed significantly reduced growth and stability of neuromuscular synapses, and impaired glutamatergic neurotransmission in $d A c s l$ mutants. The axonal aggregates and synaptic defects in $d A c s l$ mutants were fully rescued by neuronal expression of human ACSL4, supporting a functional conservation of ACSL4 across species in the nervous system. Together, our findings demonstrate that dAcsl regulates axonal transport of synaptic vesicles and is required for synaptic development and function. Defects in axonal transport and synaptic function may account, at least in part, for the pathogenesis of ACSL4-related mental retardation.

\section{Introduction}

Mental retardation (MR) is present in $1-3 \%$ of the general population and is a complex phenotype characterized by suboptimal brain function with onset before the age of 18 years (Vaillend et al., 2008; Humeau et al., 2009). MR is divided into syndromic and nonsyndromic forms, depending on whether cognitive dysfunction is associated with other anomalies. Approximately $300 \mathrm{MR}$ genes have so far been identified (Inlow and Restifo, 2004; Humeau et al., 2009). Mutations in the acyl-CoA synthetase longchain family member 4 (ACSL4), also known as long-chain fatty acyl-CoA ligase 4, result in nonsyndromic X-linked MR (Meloni et al., 2002; Longo et al., 2003). ACSL4 belongs to a family of enzymes that convert long-chain fatty acids to acyl-CoAs that

\footnotetext{
Received June 25, 2010; revised Nov. 22, 2010; accepted Dec. 2, 2010.

This work was supported by the Key Laboratory of Developmental Genes and Human Disease, Southeast University, Nanjing, and grants from the National Natural Science Foundation of China (30930033), the Chinese Academy of Sciences (KSCX2-EW-R-05), and the Ministry of Science and Technology of China (2007CB947200) to Y.Q.Z. We thank W. M. Saxton, H. Keshishian, E. Hafen, H. Krämer, T. Uemura, D. L. Deitcher, M. B. O'Connor, and H. Bellen for stocks and antibodies; and Drs. J. Liu, F. Huang, M. Ding, C. Collins, W. M. Saxton, A. Bowman, and Z. Wang for helpful discussions.

Correspondence should be addressed to Dr. Yong Q. Zhang, Institute of Genetics and Developmental Biology, Chinese Academy of Sciences, No. 1 Datun Road, Chao Yang District, Beijing 100101, China. E-mail: yqzhang@genetics.ac.cn.

DOI:10.1523/JNEUROSCI.3278-10.2011

Copyright $\odot 2011$ the authors $\quad 0270-6474 / 11 / 312052-12 \$ 15.00 / 0$
}

participate in multiple biosynthetic, metabolic, and cellular signaling pathways (Coleman et al., 2002). The enzyme is highly expressed in the hippocampus, a structure critical for learning and memory, where it is required for dendritic spine formation (Cao et al., 2000b; Meloni et al., 2002, 2009). The mechanisms by which mutations of ACSL4 lead to MR, however, are still poorly understood.

To understand the neuronal functions of ACSL4 and the pathogenesis of ACSL4-related MR, we characterized mutants of $d A c s l$, the Drosophila homolog of human ACSL4, and found that $d A c s l$ is required for the production of the morphogen decapentaplegic [Dpp, a bone morphogenetic protein (BMP)-like molecule] and for axonal targeting in the Drosophila brain (Zhang et al., 2009). These $d A c s l$ mutant phenotypes could be effectively rescued by human ACSL4, demonstrating a functional homology of Drosophila dAcsl and human ACSL4 (Zhang et al., 2009). In the present study, we report that the axons of Drosophila dAcsl mutants display distally biased axonal accumulation of heterogeneous membranous organelles resembling retrograde cargos destined for degradation, suggesting a retrograde transport defect. Live imaging of green fluorescent protein (GFP)-tagged organelles confirmed that $d A c s l$ mutations specifically impaired retrograde transport of synaptic vesicles (SVs), while the transport of mitochondria was normal. Axonal transport has been extensively studied over the last 2 decades (Duncan and Goldstein, 
2006; De Vos et al., 2008), but this study provides the first experimental evidence implicating an MR-linked gene in axonal transport of SVs.

Neuronal function is maintained by well regulated intracellular trafficking. A diverse array of newly synthesized proteins and lipids are transported anterogradely to distal synapses, while neurotrophic signals and damaged proteins are retrogradely transported back to the cell body (Chevalier-Larsen and Holzbaur, 2006; Goldstein et al., 2008). Mutations in genes involved in this bidirectional transport often cause defects in synaptic development and function. For example, mutations in motor proteins cause synaptic dystrophy and reduced neurotransmission at neuromuscular junctions (NMJs) (Gho et al., 1992; Hurd and Saxton, 1996; Pack-Chung et al., 2007; Barkus et al., 2008). As expected, dAcsl mutants show prominent synaptic atrophy with concomitantly compromised neurotransmission at NMJ terminals. Together, our findings demonstrate that $d A c s l$ regulates axonal transport of SVs and is required for synaptic growth and transmission.

\section{Materials and Methods}

Drosophila strains and genetics. The Drosophila melanogaster strain $w^{1118}$ was used as the wild-type control unless indicated otherwise. The UAS-ACSL4-Myc and a dAcsl hypomorphic allele $d A c s l^{05847}$ with a $p$ element inserted in the first intron of $d A c s l$ were described previously (Zhang et al., 2009). A $d A c s l$-null allele, $d A c s l^{K O}$, was generated by flippase-recognition target-mediated deletion between $\mathrm{PBac}\{\mathrm{RB}\}$ $A c s l^{e 02676}$ and $\mathrm{PBac}\{\mathrm{WH}\} A c s \mathrm{f}^{02764}$, removing most of the exons according to a procedure described by Parks et al. (2004) and Thibault et al. (2004). Other stocks include UAS-mito-GFP from W. M. Saxton (University of California, Santa Cruz, CA) (Pilling et al., 2006), UAS-SyteGFP (III) (Zhang et al., 2002), UAS-RFP-Atg8 from E. Hafen (Swiss Federal Institute of Technology, Zürich, Switzerland) (Köhler et al., 2009), UAS-LAMP1-GFP from H. Krämer (University of Texas Southwestern Medical Center, Dallas, TX) (Pulipparacharuvil et al., 2005), elav-GeneSwitch from H. Keshishian (Yale University, New Haven, CT) (Osterwalder et al., 2001), the motoneuron-specific OK6-Gal4 from M. B. O'Connor (University of Minnesota, St. Paul, MN), D42-Gal4 from W. M. Saxton, and the ubiquitous Tub-Gal4 from the Bloomington Stock Center (Indiana University, Bloomington, IN). For GeneSwitch rescue experiments, $d A c s l^{05847} / d A_{c s}{ }^{K O}$; elav-GeneSwitch/UAS-ACSL4$M y c$ mutant larvae at $2.5 \mathrm{~d}$ after egg laying (AEL) were transferred to food containing RU486 (mifepristone; Sigma) at a final concentration of 0.5 mu to induce neuronal expression of human ACSL4-Myc following the original protocol (Osterwalder et al., 2001) and then were dissected at $5 \mathrm{~d}$ AEL to examine axonal aggregates.

Immunohistochemical analyses and confocal microscopy. Immunostaining and confocal microscopy of larval preparations were performed as described previously (Jin et al., 2009; Liu et al., 2010). The following monoclonal antibodies were obtained from the Developmental Studies Hybridoma Bank: anti-CSP (cysteine-string protein) (6D6; 1:500), antisynapsin (3C11; 1:300), anti-Bruchpilot (Brp) (1:50), anti-Fas II (fasciclin II) (1D4; 1:50), anti-choline acetyltransferase (ChAT) (4B1; 1:1000), and anti-Myc (9E10; 1:200). Other antibodies include anti-synaptotagmin (Syt) (1:1000) and anti-Hrs (hepatocyte growth factor-regulated tyrosine kinase substrate) from H. Bellen (Baylor College of Medicine, Houston, TX) (1:1000; Lloyd et al., 2002), anti-Dlic from T. Uemura (Kyoto University, Kyoto, Japan) (1:500) (Satoh et al., 2008), antisynaptosomal-associated protein 25 (SNAP-25) from D. L. Deitcher (Cornell University, Ithaca, NY) (1:200) (Rao et al., 2001), FITCconjugated anti-horseradish peroxidase (HRP) (1:200; Jackson ImmunoResearch), and rabbit polyclonal cleaved caspase 3 (Asp175) antibody (1:100; Cell Signaling Technology). All primary antibodies were visualized using specific secondary antibodies conjugated to Alexa 488 or Alexa 568 (1:1000; Invitrogen). All images were collected using a Leica SP5 laser scanning confocal microscope and processed with Adobe Photoshop 8.0.

For quantification of axonal traffic jams, the number and total area of CSP-positive aggregates in the defined regions of one to two nerves from each animal were quantified using ImageJ. The anterior segmental nerves were defined as the region directly posterior to the larval ventral nerve cord (VNC) that spanned abdominal segments A2-A3. The axonal bundles covering the abdominal segments A6-A7 were defined as the posterior nerves. For each genotype, at least six larval preparations were analyzed.

Methods for the morphological analysis of NMJs were described previously (Jin et al., 2009; Yao et al., 2011). Briefly, serial confocal images of NMJ4 on the abdominal segment A6 were maximally projected. Individual boutons were identified according to the discrete staining signals of the synaptic vesicle marker anti-CSP. The synaptic area was determined by measuring the area of anti-CSP staining over the whole synaptic terminal with ImageJ.

Time-lapse imaging of Syt-enhanced GFP-tagged vesicles in larval motor axons. Wandering third-instar larvae were dissected in chilled HL6 solution supplemented with $4 \mathrm{~mm} \mathrm{L-glutamate}$ and $0.6 \mathrm{mM} \mathrm{CaCl}_{2}$ according to published protocols (Louie et al., 2008; Yao et al., 2011). A dissected larva was transferred to a slide, covered with a large coverslip, and immersed in fresh HL6 solution. It was then viewed at room temperature with a Leica SP5 confocal microscope. Serial images were captured every $1 \mathrm{~s}$ on a single plane for no more than $5 \mathrm{~min}$ and completed within $15 \mathrm{~min}$ after dissection.

Compiled images were opened with ImageJ, and the stacks were resliced and projected to make kymographs. Lines were drawn by hand to better illustrate the movement of individual GFP-labeled puncta in kymographs. Data analysis of live images followed the methods described by Miller et al. (2005) and Yao et al. (2011). To quantify the flux, movies were opened with ImageJ and animated at 10 frames per second. The number of GFP puncta that crossed a selected point of axon was counted and then divided by the time $(<5 \mathrm{~min})$ of observation. The average velocity of moving puncta and the percentage of puncta with stops were also statistically analyzed. Puncta moving with velocities $<0.1 \mu \mathrm{m} / \mathrm{s}$ for $>10$ s were defined as puncta with stops.

Electron microscopy. Electron microscopy (EM) of larval segmental nerves was performed largely according to a previously described procedure (Hurd and Saxton, 1996). Briefly, dissected larvae were fixed overnight at $4^{\circ} \mathrm{C}$ in $2 \%$ glutaraldehyde and $2 \%$ paraformaldehyde in $0.1 \mathrm{M}$ cacodylate buffer, $\mathrm{pH}$ 7.4. Fixed tissue was then rinsed in cacodylate buffer. Postfixing was performed in $0.5 \% \mathrm{OsO}_{4}$ and $0.8 \% \mathrm{~K}_{3} \mathrm{Fe}(\mathrm{CN})_{6}$ in cacodylate buffer for $90 \mathrm{~min}$ at room temperature. Samples were washed and stained in saturated aqueous uranyl acetate for $2 \mathrm{~h}$ on ice, dehydrated in a graded acetone series, and embedded in Spurr resin (Electron Microscopy Sciences). Ultrathin $(70-80 \mathrm{~nm})$ cross sections of axonal bundles were cut with a Leica UC6 ultramicrotome. Grids were poststained with saturated uranyl acetate for $20 \mathrm{~min}$, followed by staining with Reynold's lead citrate for 2-3 min. Processed samples were observed under a JEOL 1400 electron microscope.

Electrophysiology. Electrophysiological recordings were performed essentially as described previously (Jin et al., 2009; Wang et al., 2010). Modified HL3 saline contained $70 \mathrm{~mm} \mathrm{NaCl}, 5 \mathrm{~mm} \mathrm{KCl}, 10 \mathrm{~mm} \mathrm{MgCl}, 10$ mм $\mathrm{NaHCO}_{3}, 115 \mathrm{~mm}$ sucrose, $5 \mathrm{~mm}$ trehalose, 5 mм HEPES, pH 7.2, and $1 \mathrm{mM} \mathrm{CaCl}_{2}$. Data were analyzed only from recordings with resting potentials $<-65 \mathrm{mV}$ and input resistances $>6 \mathrm{M} \Omega$. Quantal content was defined as excitatory junction potential (EJP)/miniature EJP (mEJP) amplitudes after correcting for nonlinear summation.

Statistical analysis. All the data are expressed as the mean \pm SEM. Statistical significance in two-way and multiple-group comparisons was determined by Student's $t$ test and one-way ANOVA with Tukey's post hoc test, respectively. No asterisk denotes $p>0.05$; ${ }^{\star}$ indicates $p<0.05$; ${ }^{* *}$ denotes $p<0.01 ;{ }^{* * *}$ indicates $p<0.001$.

\section{Results}

\section{dAcsl mutants show distally biased axonal aggregates}

As the $d A c s l$ null mutants were early larval lethal, we used a transallelic combination of a null allele, $d A c s l^{K O}$, and a hypomorph allele, $d A c s l^{05847}$, which survived to pharates. In the hypomorphic $d A c s l$ mutant larvae, the most salient abnormality was the accumulation of the synaptic vesicle-associated protein CSP in axons, 
a phenotypic hallmark of defective axon transport (Hurd and Saxton, 1996; Martin et al., 1999), whereas wild-type axons showed no apparent CSP-positive aggregates (Fig. $1 A-B^{\prime}$ ). The number of CSPpositive aggregates in the anterior axons was greater in $d$ Acsl mutants (18.36 \pm 4.03 per $100-\mu \mathrm{m}$-long axon segment) than in wild types $(1.91 \pm 0.75$ per 100 $\mu \mathrm{m}$-long axon segment; $p<0.001)$. Similarly, the number of aggregates observed in the posterior axons was higher in $d A c s l$ mutants than in wild types $(34.53 \pm 5.50$ vs $3.23 \pm 1.08$ per $100-\mu \mathrm{m}$-long axon segment, $p<0.001$ ) (Fig. $1 F$ ). The area of CSP-positive aggregates in both anterior and posterior axons was also significantly greater compared with the wild types; for the posterior axons, the area of CSPpositive aggregates was $17.38 \pm 4.17 \mu \mathrm{m}^{2}$ per 100- $\mu \mathrm{m}$-long axons in $d$ Acsl mutants versus $2.33 \pm 0.55 \mu \mathrm{m}^{2}$ per $100-\mu \mathrm{m}$-long axons in wild types $(p<0.001)$ (Fig. $1 G)$. Similar aggregates were also observed in other hypomorphic mutants $d A c s l^{K O}$ / $d A c s l^{\text {sh0465 }}, d A c s l^{05847} / d A c s l^{\text {sh0465 }}, d A c s l^{05847}$ / $D f(2 R) H 3 E 1$, and $d A c s l^{\text {sh0465 } / D f(2 R) H 3 E 1 ~}$ (the $D f(2 R) H 3 E 1$ deficiency completely removes $d A c s l$ ) (data not shown), indicating that the CSP-positive aggregates were caused by $d A c s l$ hypomorph mutations. Given that the $d A c s l$ mutant phenotypes can be rescued by human ACSL4 (Zhang et al., 2009), we tested whether the axonal aggregates in $d A c s l$ mutants could be rescued by human ACSL4. Indeed, the abnormal CSP-positive aggregates in $d A c s l$ mutants were fully rescued by the ubiquitous expression of human ACSL4 driven by Tub-Gal4 (Fig. $1 C, F, G$ ), confirming that Drosophila dAcsl and human ACSL4 are functionally conserved proteins that regulate the axonal distribution of CSPpositive vesicles.

There was a strong tendency for the synaptic cargos to accumulate in the posterior nerves (Fig. $1 B, B^{\prime}, F, G$ ). But we do not know whether the aggregates are present in the proximal or distal axons, due to the fact that the segmental nerves contain 60-80 parallel axons in opposite directions of sensory neurons and motoneurons (Hurd and Saxton, 1996). The cholinergic sensory neurons project anteriorly from the peripheral cell bodies into the VNC, whereas the motoneurons project in the opposite direction from cell bodies in the VNC toward the posterior and periphery to form NMJ. To determine whether the aggregates were present in the axons of sensory neurons, motoneurons, or both, we expressed enhanced GFP (eGFP)-tagged
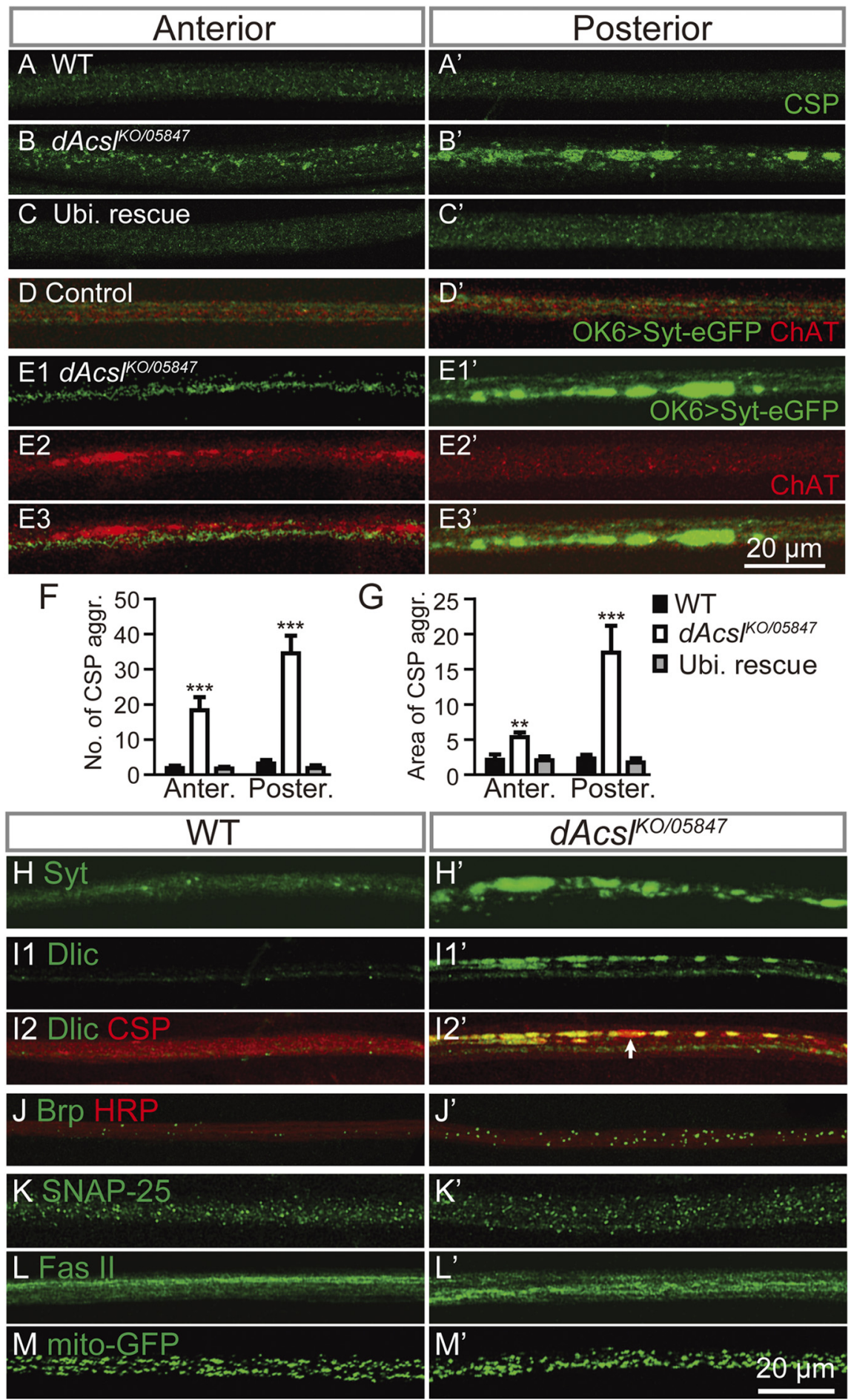

Figure 1. Distally biased axonal aggregates of synaptic cargos in $d A c s /$ mutants. $A-C^{\prime}$, Immunostaining of larval segmental nerves with

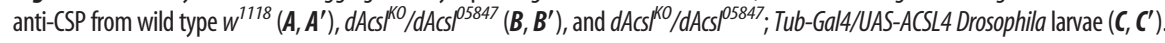
Images of axons in the area proximal to the ventral nerve cord (Anterior, $\boldsymbol{A}-\mathbf{E 3}$ ) and distal to the ventral cord (Posterior, $\boldsymbol{A}^{\prime}-\mathbf{E \mathbf { 3 } ^ { \prime }}$ ) are shown. The axonal aggregates in $d A c s /$ mutants $\left(\boldsymbol{B}, \boldsymbol{B}^{\prime}\right)$ were completely rescued by the ubiquitous expression of human ACSL 4 driven by Tub-Gal4 $\left(C, C^{\prime}\right)$.D-E3', Double labeling of axons with Syt-eGFP driven by motoneuron-specific OK6-Gal4 and anti-ChAT in the segmental nerves of control OKG-Gal4/+; UAS-Syt-eGFP/+ $\left(\boldsymbol{D}, \boldsymbol{D}^{\prime}\right)$ and mutant $d A c s{ }^{K O} / d A c s{ }^{05847}$ OK6-Gal4; UAS-Syt-eGFP/+ larvae (E1-E3'). In dAcs/ mutants, Syt-eGFP accumulations were much more prominent in the posterior axons than that in the anterior axons (compare E1' ${ }^{\prime}, \mathbf{E 1}$ ). In contrast, ChAT-positive aggregates were observed more often in the anterior axons (distal axons of sensory neurons; compare E2, E2'), indicative of distally biased axonal accumulations in both motoneurons and sensory neurons. $F, G, Q$ uantification of the number $(\boldsymbol{F})$ and the $\operatorname{area}(\boldsymbol{G})$ of CSP-positive accumulations per 100- $\mu \mathrm{m}$-long axons. $n=6$ for each genotype; ${ }^{* *} p<0.01,{ }^{* * *} p<0.001$ by one-way ANOVA with Tukey post hoc test; error bar indicates SEM. $\boldsymbol{H}-\boldsymbol{M}^{\prime}$, Posterior segmental nerves of third-instar larvae of wild type (WT) $(\boldsymbol{H}-\boldsymbol{M})$ and $d A c s{ }^{K 0} /$ dAcs ${ }^{05847}$ mutants $\left(\boldsymbol{H}^{\prime}-\boldsymbol{M}^{\prime}\right)$ stained with antibodies against $S y t\left(\boldsymbol{H}, \boldsymbol{H}^{\prime}\right)$, Dlic $(\boldsymbol{I 1}-\boldsymbol{I 2})$, and Brp $\left(\boldsymbol{J}, \boldsymbol{J}^{\prime}\right)$ showed axonal aggregates in dAcs/ mutants. The axonal distribution of SNAP-25 $\left(K, K^{\prime}\right)$, Fas $I I\left(L, L^{\prime}\right)$, and mito-GFP-labeled mitochondria $\left(M, M^{\prime}\right)$ was unaltered in $d A c s /$ mutants. Anter., Anterior; Poster., posterior; Ubi., ubiquitous; aggr., aggregates. 

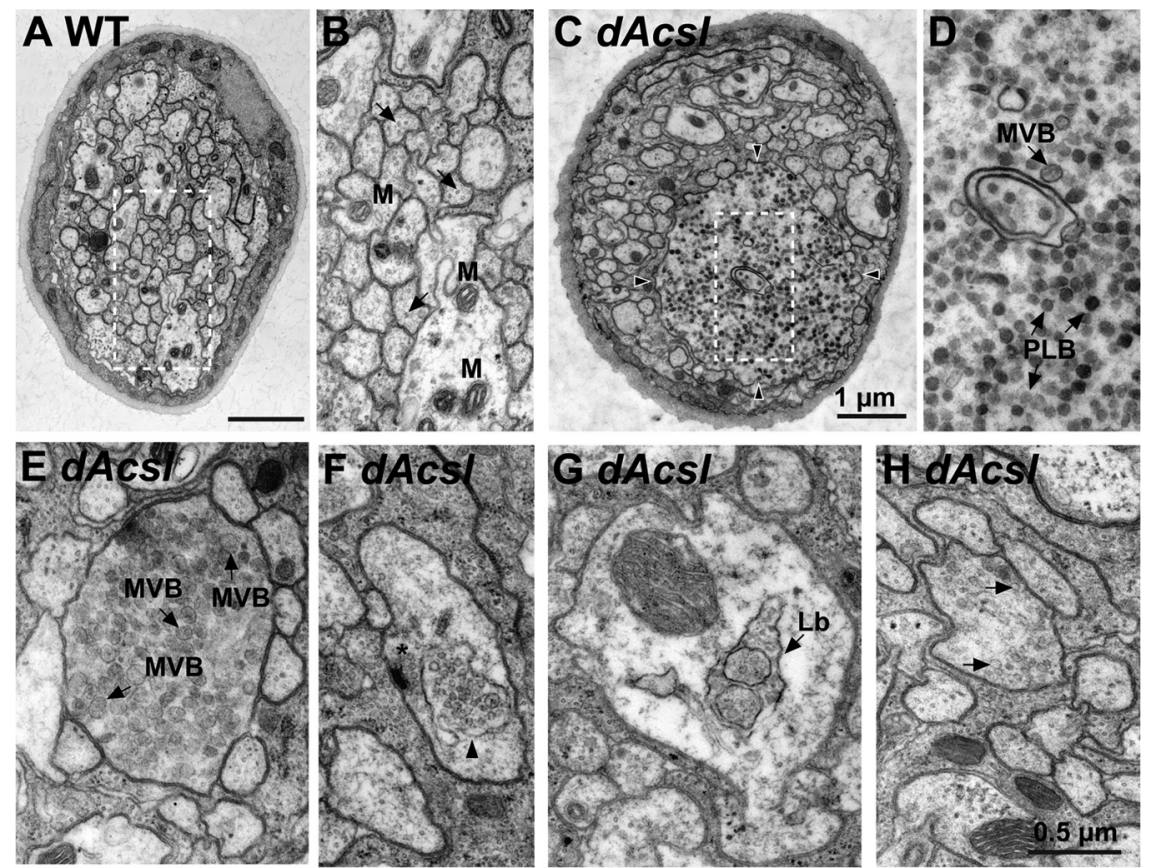

Figure 2. Accumulation of membranous organelles in $d A c s /$ mutant axons. $\boldsymbol{A}$, Micrograph of a cross section of segmental nerves from a wild-type larva. Approximately 60-80 parallel axons in opposite directions of motoneurons and sensory neurons are bundled in the central part of the nerve with the cytoplasmic processes of glial cells between some of the axons $\boldsymbol{B}, \mathbf{A}$ higher-magnification view of the boxed area in $\boldsymbol{A}$. The predominant organelles in axons are mitochondria (M) and small clear-core vesicles (arrow). C, A greatly swollen axon in a $d A c s /$ mutant (arrowheads). D, A higher-magnification view of the swollen axon in $\boldsymbol{C}$ showing MVBs and dark PLBs. $\boldsymbol{E}$, Accumulations of MVB of relatively uniform size in $d A c s /$ mutants. $\boldsymbol{F}$, An MVB with a cluster of large clear-core vesicles surrounded by a double-layer membrane in $d A$ cs/mutants (arrowhead). A putative active zone characterized by a T bar docked with vesicles is indicated by an asterisk. $\mathbf{G}, \mathrm{An} \mathrm{Lb}$ in a dAcs/ mutant axon. $\boldsymbol{H}$, Accumulations of large clear-core vesicles of different sizes (arrow) in a dAcs/mutant axon. Scale bars: $\boldsymbol{A}, \boldsymbol{C}, 1 \mu \mathrm{m}$; (in $\boldsymbol{H}$ ) $\boldsymbol{B}, \boldsymbol{D}-\boldsymbol{H}, 0.5 \mu \mathrm{m}$. itive for CSP (Fig. $1 I 1^{\prime}, I 2^{\prime}$ ), suggesting that the CSP-positive aggregates may trap dynein motors. The aggregation of Dlic could hinder retrograde transport and exacerbate the axonal aggregation. In $d A c s l$ mutants, we also observed an increased number of puncta positive for Brp, an active zone component that is transported by a vesicle subtype called Piccolo-bassoon transport vesicles (PTVs), distinct from SVs (Zhai et al., 2001; Pack-Chung et al., 2007; Goldstein et al., 2008) (Fig. 1, compare $J, J^{\prime}$ ). In contrast, immunostaining with antibodies against SNAP-25, a component of the core soluble $\mathrm{N}$-ethylmaleimidesensitive factor attachment protein (SNAP) receptor complex necessary for vesicle docking and fusion with the plasma membrane (Rao et al., 2001), and the cell adhesion molecule Fas II revealed normal distribution patterns in $d A c s l$ mutants (Fig. $1 K-L^{\prime}$ ). The number and distribution of mitochondria in axons were also normal without aggregation in $d A c s l$ mutants (Fig. $1 M, M^{\prime}$ ). Together, these data demonstrate that dAcsl mutations specifically cause aggregation of various cargos including SVs and PTVs, but the distribution of mitochondria and the plasma membrane proteins SNAP-25 and Fas II remained normal in axons. synaptotagmin (Syt-eGFP) (Zhang et al., 2002) in motoneurons under the control of OK6-Gal4. No obvious Syt-eGFPpositive aggregates were observed in wild-type axons, but many Syt-eGFP-positive aggregates of different sizes were distributed in the motoneuron axons of $d A c s l$ mutants (Fig. 1, compare E1-E3', $\left.D, D^{\prime}\right)$. The sizes of the Syt-eGFP aggregates in the distal axons of motoneurons were dramatically larger than those in the proximal axons (Fig. 1, compare E1',$E 1$ ), consistent with distally biased aggregation. ChAT is specifically expressed in cholinergic sensory neurons and is required for acetylcholine synthesis at the presynaptic bouton (Bowman et al., 1999). Accumulation of ChAT was also found in the distal axons of sensory neurons, whereas the proximal axons displayed only diffuse background staining (Fig. 1, compare E2, E2'). These data demonstrate a strong propensity for synaptic cargos to accumulate in the distal axons of both motoneurons and cholinergic sensory neurons in $d A c s l$ mutants.

To determine the specificity of the axonal aggregates in $d A c s l$ mutants, we performed immunohistochemical analysis with more antibodies and fluorescent markers. Consistent with that detected by the anti-CSP staining and Syt-eGFP marker (Fig. $1 B^{\prime}, E 1^{\prime}$ ), anti-Syt staining revealed large accumulations in the posterior axons of $d A c s l$ mutants, whereas only a few small aggregates were seen in wild types (Fig. $1 H, H^{\prime}$ ). To test whether motor proteins also accumulated in $d A c s l$ mutants, we examined the axonal distribution of the dynein light intermediate chain (Dlic), a subunit of dynein (Satoh et al., 2008), and found abundant Dlic-positive aggregates (Fig. $1 \mathrm{I1}^{\prime}$ ). Interestingly, most of the Dlic-positive aggregates were also pos-

\section{Accumulation of diverse membranous organelles in} dAcsl mutants

To further define the contents of these axonal aggregates, we examined larval segmental nerves of wild types and $d A c s l$ mutants by EM. In wild-type larvae, axons with diameters of 100-700 nm ran next to each other or were separated by the cytoplasmic processes of glial cells (Fig. 2A,B). The most common organelles in wild-type axons were mitochondria and small clear-core vesicles 20-30 $\mathrm{nm}$ in diameter (Fig. $2 \mathrm{~B}$ ). However, in the cross sections of the segmental nerves of $d A c s l^{K O} / d A c s l^{05847}$ mutants, there were conspicuous axonal swellings, up to $3 \mu \mathrm{m}$ in diameter, packed with heterogeneous membranous organelles (Fig. 2). These aggregates, most often observed in the posterior axons, include dark, prelysosomal bodies (PLBs) $60-100 \mathrm{~nm}$ in diameter (Fig. $2 C, D$ ) and multivesicular bodies (MVBs) $70-130 \mathrm{~nm}$ in diameter (Fig. $2 D, E$ ). In some mutant larvae, we also observed autophagosomes, in which a cluster of large clear-core vesicles surrounded by a double-layer membrane (Fig. $2 F$ ), and lamellated bodies (Lbs), which resemble autolysosomes (Fig. 2G). In other cases, we observed clusters of large clear-core vesicles $(40-80 \mathrm{~nm}$ in diameter) in $d A c s l$ mutant axons (Fig. $2 H$ ). These membranous aggregates (Fig. $2 \mathrm{C}-\mathrm{H}$ ) were rarely found in wild-type axons (Fig. $2 A, B$ ).

These PLBs, MVBs, and lamellated bodies could be late endosomes/lysosomes or autophagosomes. To further define the molecular and subcellular nature of these aggregates, we performed immunohistochemical analysis by double labeling. Lysosomeassociated membrane protein 1 (LAMP1)-GFP fusion protein is a commonly used late endosome/lysosome marker (Pulipparacha- 
ruvil et al., 2005). We expressed LAMP1GFP specifically in motoneurons driven by OK6-Gal4 and found prominent LAMP1-positive aggregates in $d A c s l \mathrm{mu}-$ tants (Fig. 3A1'), consistent with the accumulation of the putative PLBs observed under EM (Fig. 2). Most fluorescent signals from LAMP1-GFP overlapped with the CSP-positive aggregates (Fig. $3 A 2^{\prime}$ ). Autophagosomes can be generated locally within axons and then transported retrogradely for fusion with lysosomes (Hollenbeck, 1993; Yue, 2007). We expressed the autophagosomal marker red fluorescent protein (RFP)-Atg8 (Köhler et al., 2009) in motoneurons driven by OK6Gal4 and found obvious Atg8-positive aggregates in $d A c s l$ mutants, but no appreciable aggregates in wild types (Fig. $\left.3 B 1, B 1^{\prime}\right)$. A small portion of CSP-positive aggregates were also positive for Atg8 (Fig. $\left.3 B 2^{\prime}\right)$. The substantial colocalization of CSP, LAMP1-GFP, and RFP-Atg8 in aggregates suggests that the CSP-positive vesicles are destined for degradation by lysosomes.

The MVB marker Hrs (Lloyd et al., 2002) also accumulated, though infrequently, in the axons of $d A c s l$ mutants (Fig. $\left.3 C 1, C 1^{\prime}\right)$. Axonal MVBs are believed to carry retrogradely moving neurotrophic and/or signaling factors (Weible and Hendry, 2004). As shown in Figure $3 C$, few Hrs aggregates were positive for CSP in $d A c s l$ mutants. To better illustrate the MVB aggregates positive for Hrs, we showed an image with multiple large Hrs aggregates where only a few CSP accumulations were present (Fig. 3C2'). These results are consistent with the notion that SVs and MVBs are distinct cargos. PLBs, MVBs, and autophagosomes are normally retrograde cargos (Hirokawa et al., 1990; Quatacker et al., 1995; Hurd and Saxton, 1996). The accumulation of retrograde cargos suggests that retrograde transport might be impaired in $d A c s l$ mutants.

\section{Induced neuronal expression of human ACSL4 eliminates axonal aggregates}

To develop an ACSL4-associated MR intervention strategy, it is essential to define the in vivo spatiotemporal pattern of ACSL4 expression. We first followed the formation of axonal aggregates through larval developmental stages and found that the aggregates were formed early at the first instar larvae (2 d AEL) (Fig. $\left.4 A^{\prime}, F\right)$, indicating an early requirement for dAcsl in the normal distribution of CSP-positive vesicles in axons. Interestingly, $3.5 \mathrm{~d}$ AEL marked the transition point from slow and gradual to rapid formation of CSP-positive aggregates during larval development of $d A c s l$ mutants (Fig. $4 F$, arrow), probably due to the depletion of the maternal contribution.

We then examined whether conditional expression of ACSL4 could eliminate the already formed aggregates in $d A c s l$ mutants. We used the GeneSwitch rescue method, in which target gene expression is induced by RU486 and can be detected as early as $5 \mathrm{~h}$ later (Osterwalder et al., 2001). We first confirmed that neuronal expression of human ACSL4 driven by elav-Gal4 or OK6-Gal4 was able to rescue the axonal aggregates in $d A c s l$ mutants (data not shown), demonstrating that $d A c s l$ is cell autonomously required for the normal distribution of axonal cargos. $d A c s l^{K O}$, $d A c s l^{05847}$; elav-GeneSwitch/UAS-ACSL4-Myc mutant larvae at $2.5 \mathrm{~d}$ AEL without induction of ACSL4 showed mild CSPpositive aggregates, phenocopying the $d A c s l$ mutant phenotype (Fig. 4G). We then transferred these mutant larvae at $2.5 \mathrm{~d}$ AEL to food containing RU486 and then examined segmental nerves in wandering third instar larvae at $5 \mathrm{~d}$ AEL (Fig. $4 H$ ). After RU486 induction, all the larvae of $5 \mathrm{~d}$ AEL had appreciable levels of ACSL4 expression as detected by anti-Myc staining (Fig. $4 H$, right). The axonal aggregates observed at $2.5 \mathrm{~d}$ AEL were not observed in the RU486-treated larvae, suggesting that this aberrant phenotype was completely rescued by induced expression of ACSL4 $(p<0.001$ compared with that of larvae of $2.5 \mathrm{~d}$ AEL without RU486 treatment) (Fig. 4I). In contrast, $d A c s l$ mutants without induced expression of ACSL4 showed more and larger accumulations (Fig. $4 I$ ) at $5 \mathrm{~d}$ AEL than those examined at $2.5 \mathrm{~d}$ AEL. Induction of ACSL4 at $3 \mathrm{~d}$ AEL also partially rescued the axonal aggregates, while induction of ACSL4 at 3.5 and $5 \mathrm{~d}$ AEL did not significantly alleviate the axonal aggregates (data not shown). These results together demonstrate that induction of human ACSL4 at early larval stages can effectively eliminate the axonal aggregates in $d A c s l$ mutants.

Increased velocity of anterograde transport but reduced velocity of retrograde transport of GFP-tagged vesicles in dAcsl mutants

Axonal accumulation of synaptic cargos is often caused by transport defects (Hurd and Saxton, 1996; Martin et al., 1999; Bowman et al., 1999; Chevalier-Larsen and Holzbaur, 2006; Duncan and Goldstein, 2006; Lorenzo et al., 2010). Though the dAcsl mutants did not show the classical tail-flip phenotype of transport mutants (Bowman et al., 1999, 2000; Martin et al., 1999), the mutant larvae were sluggish with uncoordinated crawling movements (data not shown). To directly test whether $d A c s l$ is involved in axonal transport of synaptic cargos, we analyzed the dynamic behavior of Syt-eGFP-labeled vesicles in live motoneuron axons of third-instar larvae. Small mobile Syt-eGFP puncta undergo rapid bidirectional movements and could be readily visualized in 


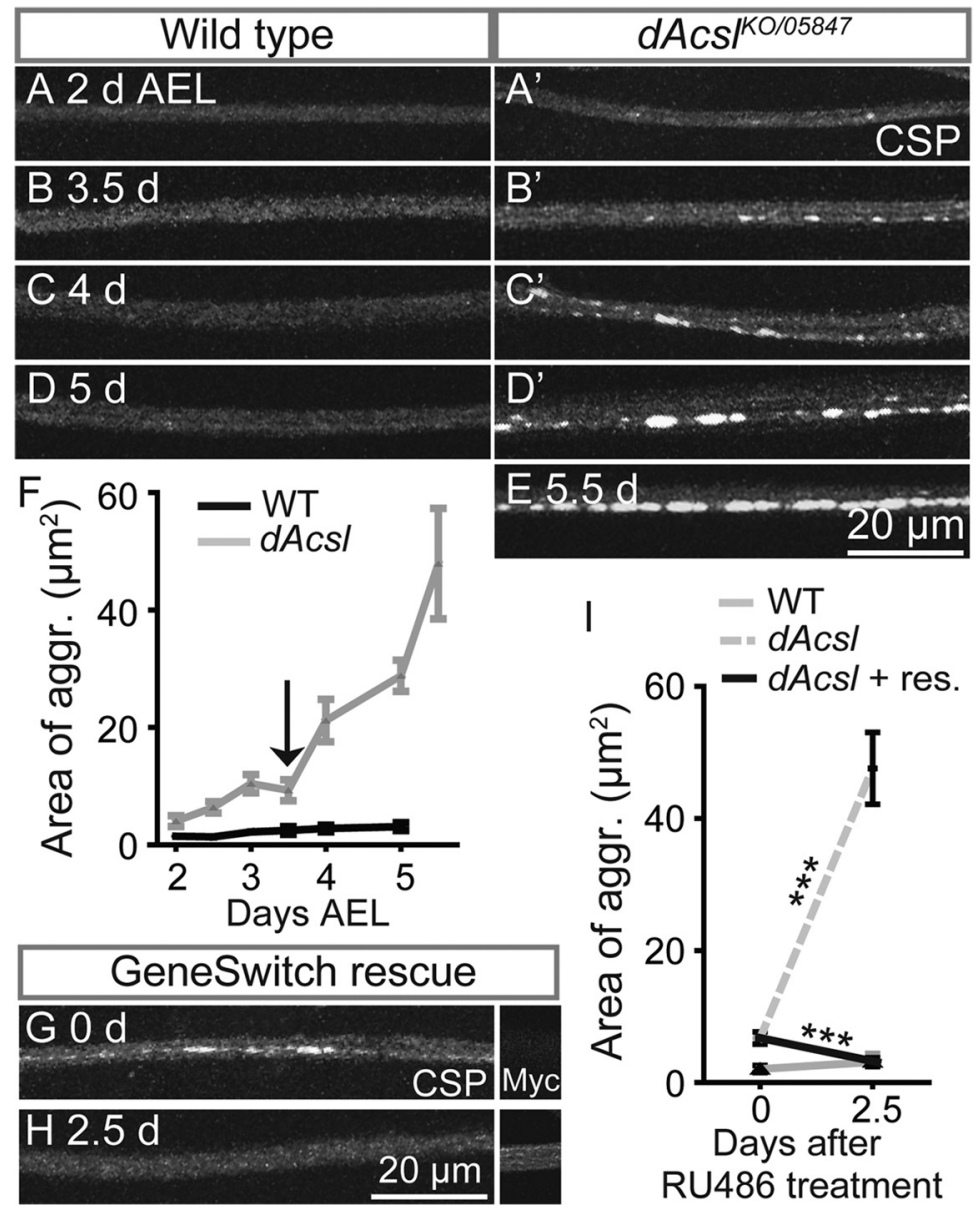

Figure 4. Induced neuronal expression of human ACSL4 eliminates axonal aggregates in $d A c s /$ mutants. $\boldsymbol{A}-\boldsymbol{E}$, Images of posterior segmental nerves of wild-type and $d A$ cs/ mutant larvae stained with anti-CSP show the axonal aggregates during larval development (in days AEL). F, Quantification of the area of axonal CSP-positive aggregates per 100 - $\mu \mathrm{m}$-long axons during larval development. The arrow indicates a transition point from slow to rapid formation of aggregates in $d A c s /$ mutants. $\boldsymbol{G}, \boldsymbol{H}$, Induced neuronal expression of human ACLS4 eliminates already formed axonal aggregates. Neuronal expression of human ACLS4-Myc can be triggered by elav-GeneSwitch in the presence of RU486. dAcs/ mutants containing the GeneSwitch machinery $(d A c s)^{K O}$ / $d A c s{ }^{\text {05847 }}$; elav-GeneSwitch/UAS-ACSL4-Myc) at $2.5 \mathrm{~d}$ AEL were exposed to RU486-containing food to induce expression of ACSL4Myc $(\boldsymbol{G})$ and then dissected at $5 \mathrm{~d}$ AEL $(\boldsymbol{H})$. The panels on the right of $\boldsymbol{G}$ and $\boldsymbol{H}$ show segmental nerves stained with anti-Myc to monitor the expression levels of human ACSL4. I, Quantification of the area of CSP-positive aggregates per 100- $\mu \mathrm{m}$-long axons. 0 and 2.5 indicate the number of days of larvae at $2.5 \mathrm{~d}$ AEL treated with RU486. $n=9$ for each genotype; ${ }^{* * *} p<0.001$ by Student's $t$ test; error bar indicates SEM. aggr., aggregates; res., rescue.

axons (supplemental Movie S1, available at www.jneurosci.org as supplemental material). Representative kymographs of axonal transport of Syt-eGFP-labeled vesicles are shown in Figure $5 A-D$. There were more stationary Syt-eGFP-positive aggregates in $d A c s l$ mutant axons (Fig. 5, compare $B, A$; vertical lines indicate stationary aggregates), consistent with the large accumulation of SVs in axons. To examine the effects of $d A c s l$ mutations on forward and reverse transport, we analyzed the behavior of individual moving particles. Anterograde flux was not significantly altered $(5.28 \pm 0.27 \mathrm{puncta} / \mathrm{min}$, for wild types vs $4.29 \pm 0.43$ puncta/min for mutants; $p=0.058$ ) (Fig. $5 E$ ). However, retrograde flux was significantly reduced in $d$ Acsl mutants $(5.34 \pm 0.25$ puncta/min for wild types vs $4.06 \pm 0.24$ per min for $d A c s l \mathrm{mu}$ tants; $p<0.01$ ) (Fig. 5C-E).

The mean velocity of anterograde moving vesicles, however, was significantly increased $[26.65 \pm 0.69 \mu \mathrm{m} / \mathrm{min}(n=687$ puncta) for control vs $31.19 \pm 0.64 \mu \mathrm{m} / \mathrm{min}$ ( $n=887$ puncta) for $d$ Acsl mutants; $p<0.001]$. Conversely, retrograde velocity was significantly reduced in $d A c s l$ mutants $[29.59 \pm 0.54 \mu \mathrm{m} / \mathrm{min}$
( $n=708$ puncta) for control vs $21.83 \pm$ $0.36 \mu \mathrm{m} / \mathrm{min}$ ( $n=1012$ puncta) for $d A c s l$ mutants; $p<0.001$ ] (Fig. $5 F, G$ ). The movement of puncta in $d A c s l$ mutants was frequently interrupted by pauses and reverses (Fig. 5D, arrows). We quantified the percentage of moving GFP-tagged vesicles with stops (velocity $<0.1 \mu \mathrm{m} / \mathrm{s}$ for $\geq 10 \mathrm{~s}$ ). No significant difference was found in the percentage of vesicles with stops moving in the anterograde direction between wild types and $d A c s l$ mutants (Fig. 5C, D, H). However, the percentage of vesicles with stops moving in the retrograde direction was significantly increased in $d A c s l$ mutants $[1.07 \pm 0.05 \%(n=9)$ for wild types vs $17.88 \pm 2.94 \%(n=9)$ for $d$ Acsl mutants; $p<0.001$ ] (Fig. $5 C, D, H$; supplemental Movie $\mathrm{S} 1$, available at www.jneurosci.org as supplemental material). These results demonstrate that the processivity of retrograde movement of Syt-eGFP-labeled SVs was compromised in $\mathrm{dAcsl}$ mutants.

The decreased retrograde processivity may cause an increase in puncta size due to a defect in the formation of membrane organelles. We then statistically analyzed the size of all moving puncta with different intensities and found no statistical difference in the retrograde puncta size between $d A c s l$ mutants and wild types (see supplemental Fig. S2, available at www. jneurosci.org as supplemental material). We also found no association between the size and the speed of retrograde moving puncta (i.e., the velocity was not dependent on the puncta size in both mutants and wild types) (supplemental Fig. S2, available at www.jneurosci.org as supplemental material). These results further support a specific transport defect in $d A c s l$ mutants.

To address whether $d A c s l$ affects transport of a specific type of cargo, or all cargos nonspecifically, we analyzed mitochondrial transport in motoneurons. Statistically, there were no differences between $d A c s l$ mutants and wild-type controls in the various transport parameters we examined (supplemental Movie S1, supplemental Fig. S1, available at www. jneurosci.org as supplemental material), suggesting that the effect of $d A c s l$ on axonal transport is specific for SVs. In summary, $d A c s l$ mutations specifically increased the velocity of anterograde transport but reduced the flux, the velocity, and the processivity of retrograde transport of SVs.

\section{dAcsl mutations substantially impair the development of NMJ synapses}

It has been reported that mutants with axonal transport defects show undergrown NMJ synapses with reduced neurotransmission (Gho et al., 1992; Hurd and Saxton, 1996; Pack-Chung et al., 2007; Barkus et al., 2008; Toda et al., 2008; Lorenzo et al., 2010). The axonal aggregates and transport defects in $d A c s l$ mutants prompted us to examine whether $d A c s l$ mutations led to abnor- 

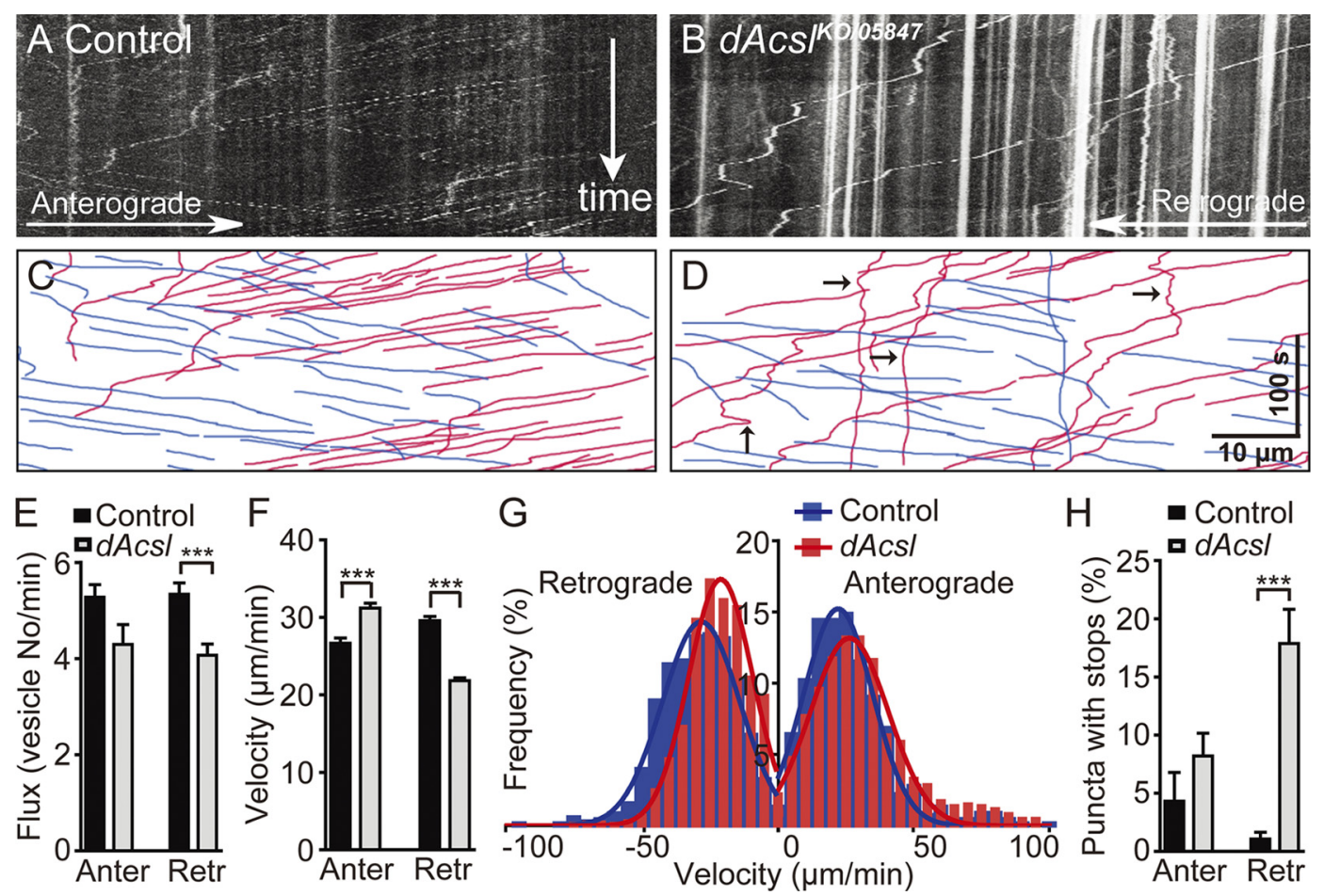

Figure 5. Altered axonal transport of GFP-tagged vesicles in both directions in $d A c s /$ mutants. $\boldsymbol{A}, \boldsymbol{B}$, Representative kymographs of Syt-eGFP-labeled SVs in motoneuron axons of heterozygous

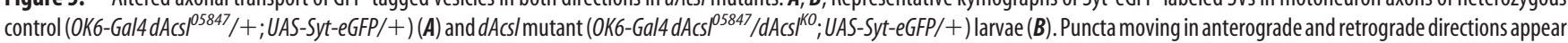
as oblique lines, whereas stationary puncta appear as vertical lines. The number of stationary puncta was far higher in mutant axons. $\boldsymbol{C}, \boldsymbol{D}$, Lines drawn by hand based on the images of $\boldsymbol{A}$ and $\boldsymbol{B}$, respectively, to better illustrate vesicle movements. Anterograde movements are shown in blue and retrograde movements are shown in red. Vertical arrows indicate reverse runs; horizontal arrows denote stops $(\boldsymbol{D})$. Stationary vesicles are not depicted. $\boldsymbol{E}, \boldsymbol{F}$, Statistical analysis of flux $(\boldsymbol{E})$ and velocity $(\boldsymbol{F})$ of both anterograde and retrograde movements. $\boldsymbol{G}$, Frequency distribution of velocities of anterograde and retrograde transports. Anterograde transport is indicated by positive values, whereas retrograde transport is depicted by negative values. $\boldsymbol{H}$, Quantification of the percentage of puncta with stops longer than $10 \mathrm{~s} . n=15$ for control and 13 for mutants; 660 and 1230 min of vesicle transports were analyzed for the control and $d A c s /$ mutants, respectively; ${ }^{* * *} p<0.001$ by Student's t test; error bar indicates SEM. Anter, Anterograde; Retr, retrograde.

mal synapses. AAcsl mutants displayed pronounced dystrophy of NMJ4 in the posterior abdominal segments A6 (Fig. 6) and A7 (data not shown), while the muscle and larval size were comparable to wild types (data not shown). We quantified the number of synaptic boutons and the synaptic area of NMJ4 in segment A6 (Fig. $6 F$ ). The bouton number and the synaptic area were significantly reduced in $d A c s l$ mutants $(8.89 \pm 3.52$ boutons in $d A c s l$ mutants vs $37.22 \pm 1.57$ boutons in wild types; for synaptic area, $19.65 \pm 8.61 \mu \mathrm{m}^{2}$ in $d A c s l$ mutants vs $152.50 \pm 7.22 \mu \mathrm{m}^{2}$ in wild types; $p<0.001$ for both bouton number and synaptic area) (Fig. $6 A, B, F, G)$. These phenotypes could be fully rescued by ubiquitous expression of human ACSL4 (36.22 $\pm 1.16 \mu \mathrm{m}^{2}$ for bouton number, $165.9 \pm 9.32 \mu \mathrm{m}^{2}$ for synaptic area; $p>0.05$ for both compared with wild types) (Fig. 6C, $F, G$ ).

To determine tissue-specific requirements for $d A c s l$, we expressed human ACSL4 using tissue-specific Gal4. Neuronal expression of ACSL4 driven by elav-Gal4 restored the bouton number to wild-type levels $(42.67 \pm 2.26 ; n=17 ; p>0.05)$ (Fig. $6 D, F, G)$, while the synaptic area was even larger than that in wild types (205.20 $\pm 9.03 \mu \mathrm{m}^{2} ; p<0.001$ compared with wild types) (Fig. $6 D, F, G$ ). Furthermore, induced neuronal expression of human ACSL4 at $2.5 \mathrm{~d}$ AEL by elav-GeneSwitch fully rescued the reduced synaptic area in $d A c s l$ mutants (for synaptic area, $165.6 \pm 12.33 \mu^{2}$ in $d A c s l$ mutants with ACSL4 expression vs $155.4 \pm 7.94 \mu \mathrm{m}^{2}$ in wild types; $\left.p>0.05\right)$. Thus, neuronal expression of ACSL4 rescued the defects in both axons (Fig. 4) and NMJ terminals of $d A c s l$ mutants. In contrast, expression of ACSL4 in postsynaptic muscles driven by Mhc-Gal4 did not rescue the dystrophic NMJs $(16.38 \pm 3.28$ for bouton number;
$52.35 \pm 11.36 \mu \mathrm{m}^{2}$ for synaptic area; $n=15 ; p>0.001$ for both parameters compared with wild types) (Fig. $6 E-G$ ). Together, these results suggest that $d A c s l$ functions primarily in the presynaptic neurons to regulate synaptic growth, consistent with its important role in axonal transport.

\section{NMJ synapses retract in late larval development in dAcsl mutants}

The size of NMJ4 synapses in the posterior abdominal segments was greatly reduced in $d A c s l$ mutants (Fig. 6), suggesting that either the synapses never fully develop or grow first but then retract. To distinguish between these possibilities, we followed the NMJ synapses in $d A c s l$ mutants during larval development. In wild types, the number of boutons and synaptic area gradually increased (Fig. $7 A-D, K$ ). At $2 \mathrm{~d}$ AEL, the synaptic area in $d A c s l$ mutants was comparable to that of wild types (Fig. $7 E, K$ ), indicating that axonal pathfinding and synaptogenesis proceeded normally. However, synapse growth during 2-3.5 d AEL of $d A c s l$ mutants was markedly slower than that in wild types (Fig. $7 K$ ). From 3.5 to $5 \mathrm{~d}$ AEL, the mutant NMJ synapses apparently retracted (Fig. $7 \mathrm{~K}$ ). The bouton size decreased and the processes between the boutons were hardly visible by $6 \mathrm{~d}$ AEL (Fig. 7I, arrow), and some disappeared altogether (Fig. 7J). Interestingly, the synaptic reduction during 3.5-4 d AEL was precisely correlated with the dramatic increase in axonal aggregates (Fig. 4).

In addition to the morphological changes in $d A c s l$ mutant synapses, we also examined other structural components of the NMJ. The microtubule cytoskeleton was visualized by antibodies against the microtubule-associated protein Futsch (Roos et al., 

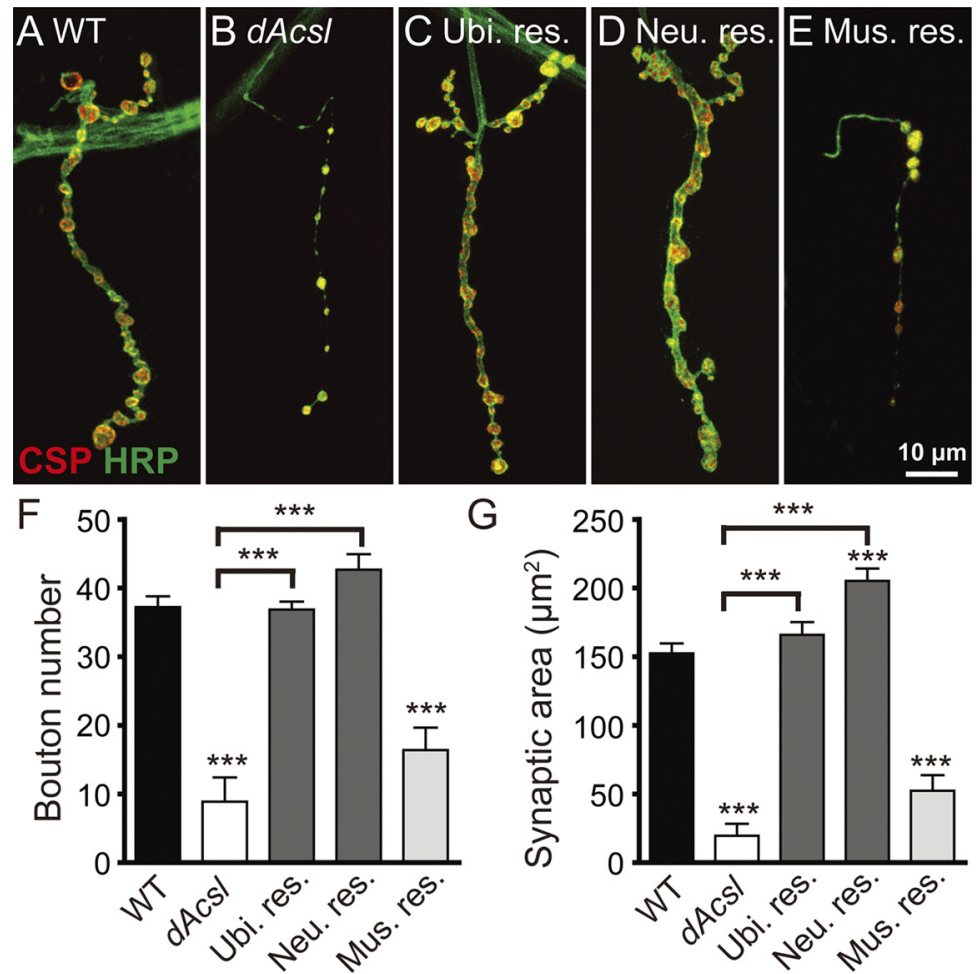

Figure 6. Presynaptic $d A c s$ is required for the normal growth of NMJ synapses. $\boldsymbol{A}-\boldsymbol{E}$, NMJ4 synapses of abdominal segment A6 were doubly stained with anti-CSP (red) and FITC-conjugated anti-HRP (green) to reveal SVs and presynaptic plasma membrane, respectively. Representative NMJs of different genotypes are shown: WT $(\boldsymbol{A}) ; d A c s /{ }^{K O} /\left.d A c s\right|^{05847}(\boldsymbol{B})$; ubiquitous rescue (Ubi. res.), $\left.d A c S\right|^{K O} / d A c s{ }^{05847} ;$ Tub-Gal4/UAS-ACSL4 (C); neuronal rescue (Neu. res.), elav-Gal4/ +;dAcs/ ${ }^{K O} / d A c s /{ }^{05847} ; U A S-A C S L 4 /+(D) ;$ and muscular rescue (Mus. res.), $\left.d A c s\right|^{K O} /\left.d A c s\right|^{55847}$; Mhc-Gal4/UAS-ACSL4 (E). In $d A c s /$ mutants $(\boldsymbol{B})$, the bouton number and synaptic area were dramatically reduced compared with the wild type $(\boldsymbol{A})$. Ubiquitous $(\boldsymbol{C})$ and pan-neuronal $(\boldsymbol{D})$ expression of human ACSL4 rescued the NMJ defects of $d A c s /$ mutants, but postsynaptic expression of ACSL4 in muscles ( $\boldsymbol{E})$ did not $(p>0.05)$. $\boldsymbol{F}, \boldsymbol{G}$, Statistical results of bouton number $(\boldsymbol{F})$ and synaptic area $(\boldsymbol{G})$ in different genotypes are presented. $n>15$ for all genotypes; ${ }^{* * *} p<0.001$ by one-way ANOVA with Tukey post hoc test; error bar indicates SEM.

2000). In wild types, the microtubule cytoskeleton was organized into a narrow core filament that extended throughout the presynaptic terminal (Fig. $7 \mathrm{~L}$ ), whereas the Futsch signal was greatly reduced or absent in $d A c s l$ mutant NMJs (Fig. $7 L^{\prime}$ ). In addition, we examined the active zone by anti-Brp staining and found that $d A c s l$ mutants showed fewer Brp-positive puncta in $d A c s l$ mutant synapses than in the controls (Fig. $7 M, M^{\prime}$ ). To determine whether the synaptic reduction was associated with the disruption of postsynaptic structures, we costained with the presynaptic marker HRP and the postsynaptic marker Dlg. There was a precise apposition of HRP with Dlg in $d A c s l$ mutants, even though the synaptic bouton was very small (Fig. $7 N, N^{\prime}$ ). In summary, there was a concomitant reduction of the number of synaptic components, including SVs, microtubule cytoskeleton, the active zone protein Brp, and the postsynaptic scaffold protein Dlg in the small synaptic terminals of $d A c s l$ mutants.

The reduction of synaptic area in $d A c s l$ mutants may be caused by neuronal death. Inhibition of ACSL 4 by a specific inhibitor in cell cultures induced arachidonic acid-induced apoptosis, whereas ACSL4 overexpression had the opposite effect (Cao et al., 2000a). To determine whether $d A c s l$ mutations affected apoptosis, we stained the VNCs of wild types and $d A c s l$ mutants with anti-cleaved-caspase 3, a marker of early apoptosis (Yu et al., 2002). Surprisingly, we found that the number of caspase 3 -positive cells in the VNC was significantly lower in $d A c s l \mathrm{mu}-$ tants $(30.67 \pm 4.63$ per VNC in wild types vs $16.2 \pm 5.38$ per VNC in $d A c s l$ mutants; $n=6$ for wild type and $n=5$ for $d A c s l ; p<$
0.05) (Fig. 7O,P), suggesting that the synapse retraction in $d A c s l$ mutants was not caused by apoptosis.

\section{$d A c s l$ is required for normal synaptic transmission}

The accumulation of synaptic cargos in axons (Figs. 1-4), the altered transport of Syt-eGFP-labeled SVs (Fig. 5), and the reduced synaptic size (Fig. 6) in $d A c s l$ mutants may compromise neuromuscular transmission. To determine whether $d A c s l$ mutants had altered neurotransmission, we examined both evoked and spontaneous synaptic glutamate release using intracellular recordings from muscle 6 in the posterior abdominal segments A6. The evoked EJPs were significantly reduced in $d A c s l$ mutants $(41.35 \pm 1.11 \mathrm{mV}$ in wild type vs $26.11 \pm 1.31 \mathrm{mV}$ in $d A c s l$ mutants; $n=11$ for wild type and $n=14$ for $d$ Acsl; $p<0.001$ ) (Fig. 8A, B,F). The mEJPs (also known as quantal size) were normal in $d A c s l$ mutants $(1.03 \pm 0.02 \mathrm{mV}$ in wild type vs $0.97 \pm 0.04 \mathrm{mV}$ in $d A c s l$ mutants; $p<0.001$ ) (Fig. $8 A, B, G$ ). Quantal content, as determined by dividing the EJP amplitudes (corrected for nonlinear summation) by average mEJP amplitudes, was significantly reduced in $d A c s l$ mutants (159.1 \pm 10.1 in wild type vs $48.9 \pm 3.8$ in $d A c s l$ mutants; $p<0.001$ ) (Fig. $8 A, B, H$ ). Thus, $d A c s l$ is required for the normal synaptic transmission.

The reduced EJPs and quantal content were fully rescued by human ACSL4 expressed either ubiquitously $(47.36 \pm 1.54$ and $175.4 \pm 16.57 \mathrm{mV}$, respectively; $p>0.05$ for both) or pan-neuronally $(45.79 \pm 1.01$ and $179.1 \pm 20.35 \mathrm{mV}$, respectively; $p>0.05$ for both) (Fig. $8 F, H)$. The EJPs in animals with ACSL4 ubiquitously expressed and mEJPs in animals with ACSL4 expressed pan-neuronally were mildly but significantly higher than that in wild types $(47.36 \pm 1.54 \mathrm{mV}$ EJP in ubiquitously expressed larvae; $1.30 \pm 0.10 \mathrm{mV} \mathrm{mEJP}$ in neuronally expressed larvae; $n=10$ for both) (Fig. $8 F, G$ ). However, postsynaptic expression of ACSL4 driven by Mhc-Gal4 in $d A c s l$ mutant background slightly rescued EJPs $(32.92 \pm 1.22 \mathrm{mV}$; $p<0.01)$ but did not rescue quantal content $(p>0.05$ compared with the mutant) (Fig. 8). These results indicate that $d A c s l$ mutations primarily disrupted presynaptic function, consistent with our results demonstrating a role for dAcsl in axonal transport (Figs. 4, 5) and NMJ development (Fig. 6).

\section{Discussion}

$d A c s l$ mutations primarily impair retrograde axonal transport of SVs

Light and electron microscopic analysis showed prominent axonal aggregates of SVs in $d A c s l$ mutants (Figs. 1-3). One possibility for the aggregate formation is a targeting or fusion defect of SVs to the synaptic terminals in $d A c s l$ mutants. However, alterations in the expressions of mammalian $\beta$-catenin and scribble, which are required for clustering SVs at presynaptic sites, result in no axonal SV aggregates (Bamji et al., 2003; Sun et al., 2009). 

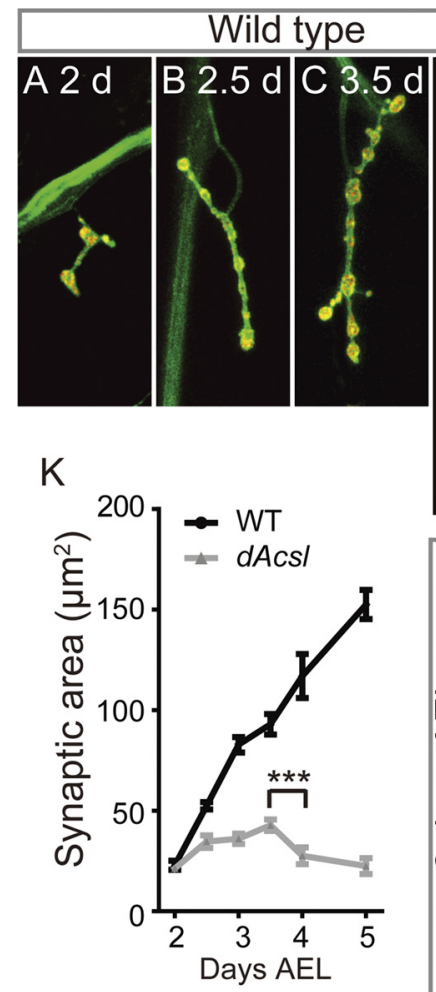
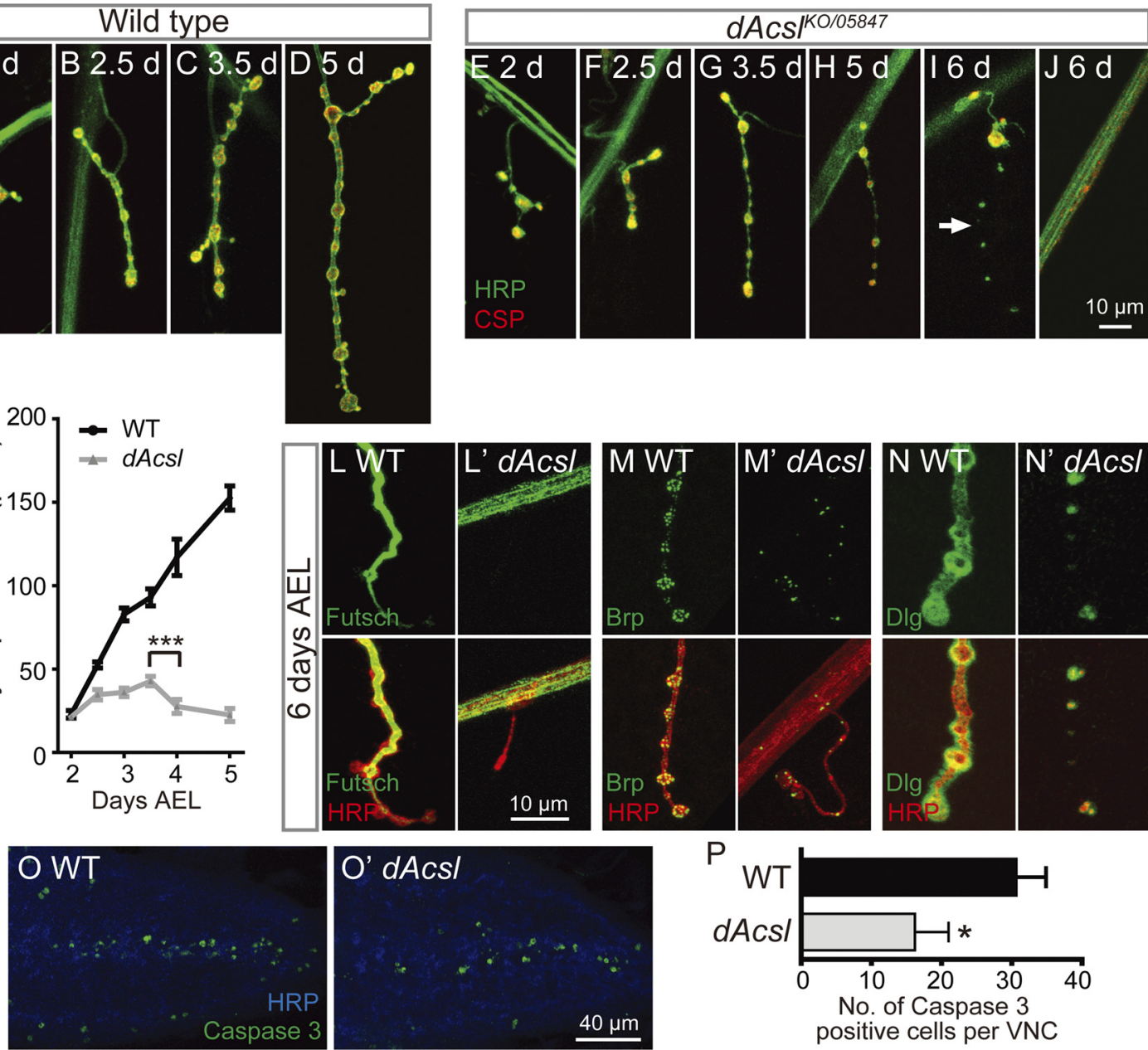

Figure 7. NMJ synapses retract during late larval development in dAcs/ mutants. $\boldsymbol{A}-\boldsymbol{J}$, NMJ4 synapses of abdominal segment A6 were labeled with anti-CSP (red) and FITC-conjugated anti-HRP ( $g r e e n)$ to reveal SVs and presynaptic membranes, respectively. The developmental stages are indicated by the number of days AEL. The synaptic span and bouton number of wild-type larvae (WT) gradually increased with development $(\boldsymbol{A}-\boldsymbol{D})$. In $d A(s /$ mutants, the NMJ synapses grow in the early stage of development until $3.5 \mathrm{~d}$ AEL but retracted afterward $(\boldsymbol{E}-\boldsymbol{J})$, even though the larva keeps growing. Two representative NMJs of $d A$ cs $/$ mutants at $6 \mathrm{~d}$ AEL are shown in $I$ and $J$. The neuronal processes revealed by anti-HRP staining between boutons are barely visible $(I$, arrow). Some of the mutants totally lost NMJs $(J)$. $K, Q$ uuantification of the synaptic area of wild-type and $d A c s /$ mutants during development. $\boldsymbol{L}-\boldsymbol{L}^{\prime}$, The staining of Futsch, which labels stable microtubule bundles in synaptic terminals was absent in $d A(s)$ mutants. $\boldsymbol{M}-\boldsymbol{M}$, , The number of puncta positive for the active zone marker anti-Brp was greatly reduced in $A$ Acs $/$ mutants. $N-N^{\prime}$, The postsynaptic marker Dlg surrounding the presynaptic membrane labeled by anti-HRP staining was

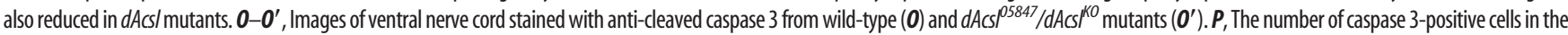
ventral nerve cord was significantly decreased in $d A c s$ mutants. $n=9$ for each genotype; ${ }^{*} p<0.05,{ }^{* * *} p<0.001$ by Student's $t$ test; error bar indicates SEM.

Similarly, mutations in Drosophila exocyst components Sec5 and Sec15 involved in the secretory pathway lead to no axonal accumulations either (Murthy et al., 2003; Mehta et al., 2005). Therefore, it is unlikely that the axonal aggregates in $d A c s l$ mutants result from a defect in SV targeting or fusion. Another possibility for the aggregates is a defect in protein turnover or the formation of membrane organelles in $d A c s l$ mutants. However, Shen and Ganetzky (2009) have recently reported that mutants of the autophagy pathway do not show axonal aggregates. Mutants of spinster, encoding a multipass transmembrane protein involved in late endosome/lysosome function (Sweeney and Davis, 2002), show accumulations of membrane structures in the soma and NMJ synapses (Dermaut et al., 2005), but no axonal aggregates (data not shown). Furthermore, we found that LAMP1-GFP, RFP-Atg8, and Hrs were localized normally in both the cell bodies and the NMJ terminals (data not shown), but accumulated specifically in the axons of $d$ Acsl mutant motoneurons (Fig. 3). Together, it seems unlikely that the axonal aggregates in $d A c s l$ mutants are caused by a defect in SV targeting, protein degradation, or the formation of membrane organelles.
Four independent lines of evidence support the idea that $d A c s l$ primarily regulates retrograde transport of SVs. First, there was a distally biased accumulation of aggregates, a phenotype shared by the mutants of roadblock, which encodes a dynein-associated protein mediating retrograde transport (Bowman et al., 1999). Consistently, motoneurons innervating the anterior muscles with shorter axons showed well developed NMJ synapses with fewer axonal aggregates (data not shown). Second, immunohistochemical analysis showed that the CSP-positive aggregates in $d A c s l$ mutants were mostly positive for the late endosome/lysosome marker LAMP1 and partly positive for the autophagosome marker Atg8. Consistently, EM analysis revealed that there were conspicuous PLBs and MVBs in $d$ Acsl mutant axons (Figs. 2, 3), reminiscent of the accumulations of retrograde cargos observed on the distal side of ligated axons (Hirokawa et al., 1990, 1991). These data demonstrated an accumulation of mostly retrograde organelles in $d A c s l$ mutants. Third, the dynein subunit Dlic also accumulated and colocalized with the CSP-positive aggregates in $d A c s l$ axons (Fig. 1). The limited availability of Dlic could inhibit the retrograde transport of CSP-positive SVs. It would be inter- 


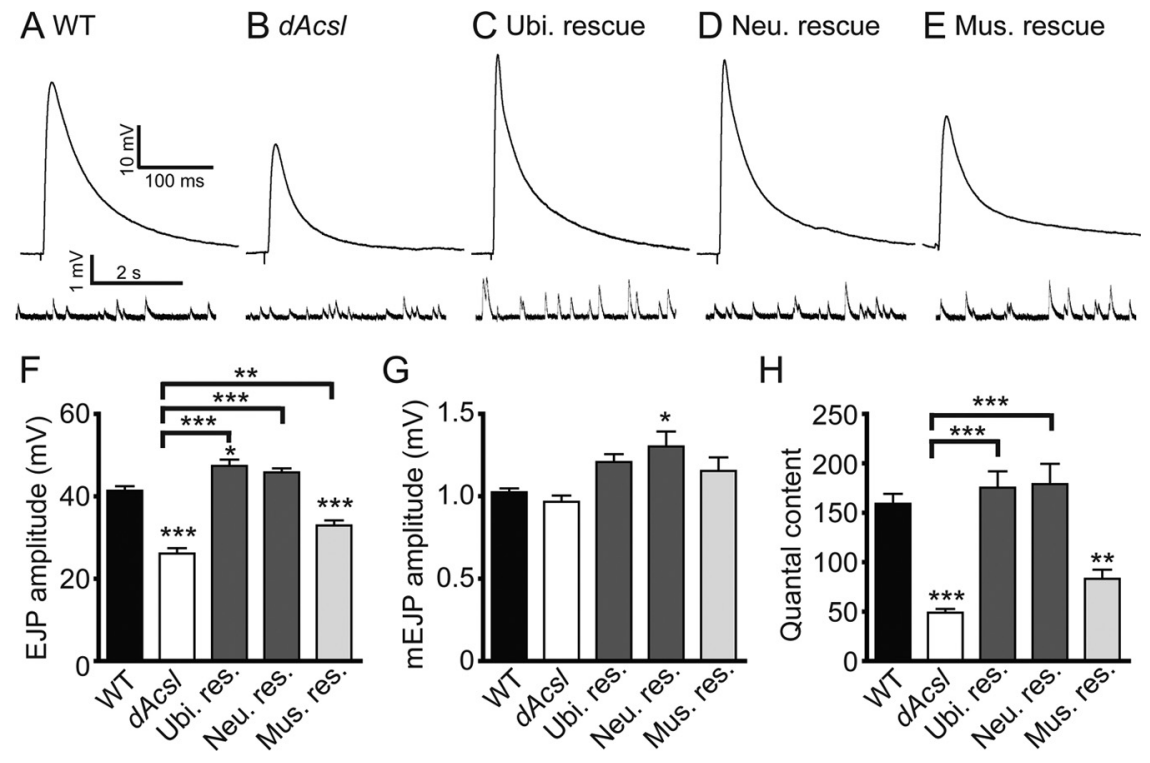

Figure 8. Reduced synaptic transmission in $d A c s /$ mutants. $\boldsymbol{A}-\boldsymbol{E}$, Representative traces of EJPs (top panels) and mEJPs (bottom panels) of NMJ6/7 from the abdominal segment A6 of WT $(\boldsymbol{A})$; $\left.d A c s\right|^{K O} /\left.d A c s\right|^{05847}(\boldsymbol{B})$; ubiquitous rescue (Ubi. res.), $\left.d A c s\right|^{K O}$ / $d A c s{ }^{05847}$; Tub-Gal4/UAS-ACSL4 (C); neuronal rescue (Neu. res.), elav-Gal4/+;dAcs/ ${ }^{K O} / d A c s{ }^{05847} ; U A S-A C S L 4 /+(D)$; and muscular rescue (Mus. res.), $\left.d A c s\right|^{K O} /\left.d A c s\right|^{05847} ;(57-G a l 4 / U A S-A C S L 4(\boldsymbol{E})$. The scales for both EJP and mEJP traces are depicted in $\boldsymbol{A}$. $\boldsymbol{F}-\boldsymbol{H}$, Statistical results of EJP amplitudes $(\boldsymbol{F}), \mathrm{mEJP}$ amplitudes $(\boldsymbol{G})$, and quantal content $(\boldsymbol{H})$ in different genotypes $(n \geq 10)$ are presented. ${ }^{*} p<0.05,{ }^{* *} p<0.01,{ }^{* * *} p<0.001$ by one-way ANOVA with Tukey post hoc test; error bar indicates SEM.

tions that reduce levels of neutral lipids (Zhang et al., 2009) could alter the lipid composition of the cargo membranes, resulting in abnormal anchorage of motors to membranous cargos. Previous studies showed that binding of dyneindynactin to the acidic phospholipids phosphatidic acid or phosphatidylinositol 4,5-bisphosphate $\left[\operatorname{PtdIns}(4,5) \mathrm{P}_{2}\right]$ in vesicle membranes requires spectrin (Muresan et al., 2001). Knockdown of spectrin or expression of a mutated spectrin in motoneurons caused axonal aggregates and slowed and erratic transport of SVs in Drosophila (Pielage et al., 2005; Lorenzo et al., 2010). It would be interesting to test whether $d A c s l$ and spectrin interact in regulating axonal transport. Alternatively, dAcsl, acting in the first step of lipid metabolism, could alter the levels of certain lipid-related signaling molecules, which in turn regulate axonal transport.

\section{dAcsl mutants show atrophic NMJ synapses}

Synaptic atrophy has been well documented in many mutants that show ax-

esting to test whether overexpression of retrograde motors such as Dlic would rescue the axonal and synaptic defects in $d A c s l$ mutants. Finally, live imaging of Syt-eGFP-tagged vesicles in motoneurons showed that the flux, velocity, and processivity of retrograde transport were all significantly reduced in $d A c s l \mathrm{mu}-$ tants (Fig. 5). Defective retrograde transport may contribute to the distally biased accumulation of the various axonal cargos.

Axonal vesicles can associate with both anterograde kinesins and retrograde dyneins, and anterograde and retrograde transport show interdependence. Two hypotheses have been proposed to explain bidirectional transport. One is the "tug-ofwar" hypothesis in which both simultaneously active anterograde and retrograde motors move the same cargo with different efficacy. The other is the "coordination" hypothesis in which additional factors inhibit one of the opposing motors for direction-selective transport (Martin et al., 1999; Gross, 2004; Welte, 2004; Müller et al., 2008). The unprecedented reduced retrograde velocity concomitant with enhanced anterograde transport of SVs in $\mathrm{AAcsl}$ mutants favors the model of tug-of-war, as the coordination model predicts a transport defect in only one direction.

How $d$ Acsl regulates axonal transport is currently unknown. The finding that transport of mitochondria was unaffected in $d A c s l$ mutants indicates that the microtubules along which mitochondria move and the energy supply are normal, though the microtubules detected by anti-Futsch staining in the synaptic terminals retract or disappear in $d A c s l$ mutants (Fig. $7 L^{\prime}$ ). There are robust genetic interactions among motors and their interacting proteins (Martin et al., 1999; Lorenzo et al., 2010). However, we found no obvious genetic interaction between $\mathrm{AAcsl}$ and motor genes such as $K h c$, roadblock, or $p 150^{\text {glued }}$ (data not shown) in regulating the axonal aggregates, suggesting that motors and their interacting proteins were not substantially compromised in $\mathrm{AAcsl}$ mutants. Still, there are at least two possible explanations for the transport defects observed in $d A c s l$ mutants. $d A c s l$ muta- onal aggregates and transport defects (Hurd and Saxton, 1996; Pack-Chung et al., 2007; Barkus et al., 2008; Toda et al., 2008; Lorenzo et al., 2010). Mounting evidence establishes that axonal transport defects lead to synapse defects, but synapse defects may not necessarily result from transport defects. In this study, we show that $d A c s l$ mutants display significantly reduced synaptic size and impaired neurotransmission, demonstrating that dAcsl is required for synaptic growth and transmission (Figs. 6-8). Though dAcsl is widely expressed in both presynaptic neurons and postsynaptic muscle cells (data not shown) (Zhang et al., 2009), it is interesting to note that dAcsl functions mainly on the presynaptic side (Figs. 6, 8). The atrophic NMJ synapses in $d A c s l$ mutants are consistent with the reduced number of dendritic spines in cultured hippocampal neurons with Acsl4 knocked down by RNA interference (Meloni et al., 2009). Importantly, the synaptic defects in $d A c s l$ mutants can be fully rescued by human ACSL4, demonstrating a conserved synaptic function between Drosophila dAcsl and human ACSL4.

Developmental analysis revealed that the NMJ synapses grow more slowly in early larval development and later retract in late larval development of $d A c s l$ mutants (Fig. 7). The synapse retraction was precisely concomitant with the rapid formation of axonal aggregates (compare Figs. $7 K, 4 F$ ), indicating that the appearance of large aggregates may destabilize the developing synapses. The synapse retraction in $d A c s l$ mutants is different from the synapse-dismantling process characterized by diffusion and degradation of postsynaptic Dlg during metamorphosis (Liu et al., 2010). It is also different from the synapse disassembly process characterized by presynaptic retraction while postsynaptic machinery remains during normal larval development (Eaton et al., 2002). However, there are at least two similarities between the synapse retraction in $\mathrm{dAcsl}$ mutants and the synapse disassembly process reported previously (Eaton et al., 2002; Eaton and Davis, 2005; Pielage et al., 2005). First, early disruption of presynaptic microtubule cytoskeleton is observed in both processes. 
Second, a defect in retrograde transport of BMP signaling may be shared by both processes. It has been well documented that a defect in retrograde BMP signaling dramatically reduces the synaptic size at Drosophila NMJs (McCabe et al., 2003). Specifically, blocking retrograde axonal transport by overexpression of a dominant-negative of $\mathrm{p} 150^{\text {Glued }}$, a component of the dynactin complex, inhibits BMP signaling and leads to dystrophic NMJs (McCabe et al., 2003). In addition to the dystrophic NMJs, mutants in the BMP signaling pathway and the dynactin complex also show enhanced disassembly and retraction of NMJ synapses (Eaton et al., 2002; Eaton and Davis, 2005). It would be interesting to test whether the synaptic defects in $d A c s l$ mutants result, at least partially, from a compromise in the BMP signaling pathway.

In summary, using a combination of confocal microscopy, EM, live imaging, and electrophysiological analysis, we demonstrate that $d A c s l$ specifically regulates axonal transport of SVs, synaptic growth, and neurotransmission. The neuronal functions of dAcsl can be substituted by human ACSL. Thus, the power of Drosophila genetics and the obvious phenotypes of $d A c s l$ mutants can be used to screen for suppressor genes or chemicals for ACSL4-associated pathologies.

\section{References}

Bamji SX, Shimazu K, Kimes N, Huelsken J, Birchmeier W, Lu B, Reichardt LF (2003) Role of beta-catenin in synaptic vesicle localization and presynaptic assembly. Neuron 40:719-731.

Barkus RV, Klyachko O, Horiuchi D, Dickson BJ, Saxton WM (2008) Identification of an axonal kinesin-3 motor for fast anterograde vesicle transport that facilitates retrograde transport of neuropeptides. Mol Biol Cell 19:274-283.

Bowman AB, Patel-King RS, Benashski SE, McCaffery JM, Goldstein LS, King SM (1999) Drosophila roadblock and Chlamydomonas LC7: a conserved family of dynein-associated proteins involved in axonal transport, flagellar motility, and mitosis. J Cell Biol 146:165-180.

Bowman AB, Kamal A, Ritchings BW, Philp AV, McGrail M, Gindhart JG, Goldstein LS (2000) Kinesin-dependent axonal transport is mediated by the sunday driver (SYD) protein. Cell 103:583-594.

Cao Y, Murphy KJ, McIntyre TM, Zimmerman GA, Prescott SM (2000a) Expression of fatty acid-CoA ligase 4 during development and in brain. FEBS Lett 467:263-267.

Cao Y, Pearman AT, Zimmerman GA, McIntyre TM, Prescott SM (2000b) Intracellular unesterified arachidonic acid signals apoptosis. Proc Natl Acad Sci U S A 97:11280-11285.

Chevalier-Larsen E, Holzbaur EL (2006) Axonal transport and neurodegenerative disease. Biochim Biophys Acta 1762:1094-1108.

Coleman RA, Lewin TM, Van Horn CG, Gonzalez-Baró MR (2002) Do long-chain acyl-CoA synthetases regulate fatty acid entry into synthetic versus degradative pathways? J Nutr 132:2123-2126.

Dermaut B, Norga KK, Kania A, Verstreken P, Pan H, Zhou Y, Callaerts P, Bellen HJ (2005) Aberrant lysosomal carbohydrate storage accompanies endocytic defects and neurodegeneration in Drosophila benchwarmer. J Cell Biol 170:127-139.

De Vos KJ, Grierson AJ, Ackerley S, Miller CC (2008) Role of axonal transport in neurodegenerative diseases. Annu Rev Neurosci 31:151-173.

Duncan JE, Goldstein LS (2006) The genetics of axonal transport and axonal transport disorders. PLoS Genet 2:e124.

Eaton BA, Davis GW (2005) LIM Kinase1 controls synaptic stability downstream of the type II BMP receptor. Neuron 47:695-708.

Eaton BA, Fetter RD, Davis GW (2002) Dynactin is necessary for synapse stabilization. Neuron 34:729-741.

Gho M, McDonald K, Ganetzky B, Saxton WM (1992) Effects of kinesin mutations on neuronal functions. Science 258:313-316.

Goldstein AY, Wang X, Schwarz TL (2008) Axonal transport and the delivery of pre-synaptic components. Curr Opin Neurobiol 18:495-503.

Gross SP (2004) Hither and yon: a review of bi-directional microtubulebased transport. Phys Biol 1:R1-R11.

Hirokawa N, Sato-Yoshitake R, Yoshida T, Kawashima T (1990) Brain dynein (MAP1C) localizes on both anterogradely and retrogradely transported membranous organelles in vivo. J Cell Biol 111:1027-1037.
Hirokawa N, Sato-Yoshitake R, Kobayashi N, Pfister KK, Bloom GS, Brady ST (1991) Kinesin associates with anterogradely transported membranous organelles in vivo. J Cell Biol 114:295-302.

Hollenbeck PJ (1993) Products of endocytosis and autophagy are retrieved from axons by regulated retrograde organelle transport. J Cell Biol 121:305-315.

Humeau Y, Gambino F, Chelly J, Vitale N (2009) X-linked mental retardation: focus on synaptic function and plasticity. J Neurochem 109:1-14.

Hurd DD, Saxton WM (1996) Kinesin mutations cause motor neuron disease phenotypes by disrupting fast axonal transport in Drosophila. Genetics 144:1075-1085.

Inlow JK, Restifo LL (2004) Molecular and comparative genetics of mental retardation. Genetics 166:835-881.

Jin S, Pan L, Liu Z, Wang Q, Xu Z, Zhang YQ (2009) Drosophila Tubulinspecific chaperone $E$ functions at neuromuscular synapses and is required for microtubule network formation. Development 136:1571-1581.

Köhler K, Brunner E, Guan XL, Boucke K, Greber UF, Mohanty S, Barth JM, Wenk MR, Hafen E (2009) A combined proteomic and genetic analysis identifies a role for the lipid desaturase Desat1 in starvation-induced autophagy in Drosophila. Autophagy 5:980-990.

Liu Z, Chen Y, Wang D, Wang S, Zhang YQ (2010) Distinct presynaptic and postsynaptic dismantling processes of Drosophila neuromuscular junctions during metamorphosis. J Neurosci 30:11624-11634.

Lloyd TE, Atkinson R, Wu MN, Zhou Y, Pennetta G, Bellen HJ (2002) Hrs regulates endosome membrane invagination and tyrosine kinase receptor signaling in Drosophila. Cell 108:261-269.

Longo I, Frints SG, Fryns JP, Meloni I, Pescucci C, Ariani F, Borghgraef M, Raynaud M, Marynen P, Schwartz C, Renieri A, Froyen G (2003) A third MRX family (MRX68) is the result of mutation in the long chain fatty acid-CoA ligase 4 (FACL4) gene: proposal of a rapid enzymatic assay for screening mentally retarded patients. J Med Genet 40:11-17.

Lorenzo DN, Li MG, Mische SE, Armbrust KR, Ranum LP, Hays TS (2010) Spectrin mutations that cause spinocerebellar ataxia type 5 impair axonal transport and induce neurodegeneration in Drosophila. J Cell Biol 189:143-158.

Louie K, Russo GJ, Salkoff DB, Wellington A, Zinsmaier KE (2008) Effects of imaging conditions on mitochondrial transport and length in larval motor axons of Drosophila. Comp Biochem Physiol A Mol Integr Physiol 151:159-172.

Martin M, Iyadurai SJ, Gassman A, Gindhart JG Jr, Hays TS, Saxton WM (1999) Cytoplasmic dynein, the dynactin complex, and kinesin are interdependent and essential for fast axonal transport. Mol Biol Cell 10:3717-3728.

McCabe BD, Marqués G, Haghighi AP, Fetter RD, Crotty ML, Haerry TE, Goodman CS, O'Connor MB (2003) The BMP homolog Gbb provides a retrograde signal that regulates synaptic growth at the Drosophila neuromuscular junction. Neuron 39:241-254.

Mehta SQ, Hiesinger PR, Beronja S, Zhai RG, Schulze KL, Verstreken P, Cao Y, Zhou Y, Tepass U, Crair MC, Bellen HJ (2005) Mutations in Drosophila sec15 reveal a function in neuronal targeting for a subset of exocyst components. Neuron 46:219-232.

Meloni I, Muscettola M, Raynaud M, Longo I, Bruttini M, Moizard MP, Gomot M, Chelly J, des Portes V, Fryns JP, Ropers HH, Magi B, Bellan C, Volpi N, Yntema HG, Lewis SE, Schaffer JE, Renieri A (2002) FACL4, encoding fatty acid-CoA ligase 4 , is mutated in nonspecific X-linked mental retardation. Nat Genet 30:436-440.

Meloni I, Parri V, De Filippis R, Ariani F, Artuso R, Bruttini M, Katzaki E, Longo I, Mari F, Bellan C, Dotti CG, Renieri A (2009) The XLMR gene ACSL4 plays a role in dendritic spine architecture. Neuroscience 159:657-669.

Miller KE, DeProto J, Kaufmann N, Patel BN, Duckworth A, Van Vactor D (2005) Direct observation demonstrates that Liprin-alpha is required for trafficking of synaptic vesicles. Curr Biol 15:684-689.

Müller MJ, Klumpp S, Lipowsky R (2008) Tug-of-war as a cooperative mechanism for bidirectional cargo transport by molecular motors. Proc Natl Acad Sci U S A 105:4609-4614.

Muresan V, Stankewich MC, Steffen W, Morrow JS, Holzbaur EL, Schnapp BJ (2001) Dynactin-dependent, dynein-driven vesicle transport in the absence of membrane proteins: a role for spectrin and acidic phospholipids. Mol Cell 7:173-183.

Murthy M, Garza D, Scheller RH, Schwarz TL (2003) Mutations in the exo- 
cyst component Sec5 disrupt neuronal membrane traffic, but neurotransmitter release persists. Neuron 37:433-447.

Osterwalder T, Yoon KS, White BH, Keshishian H (2001) A conditional tissue-specific transgene expression system using inducible GAL4. Proc Natl Acad Sci U S A 98:12596-12601.

Pack-Chung E, Kurshan PT, Dickman DK, Schwarz TL (2007) A Drosophila kinesin required for synaptic bouton formation and synaptic vesicle transport. Nat Neurosci 10:980-989.

Parks AL, Cook KR, Belvin M, Dompe NA, Fawcett R, Huppert K, Tan LR, Winter CG, Bogart KP, Deal JE, Deal-Herr ME, Grant D, Marcinko M, Miyazaki WY, Robertson S, Shaw KJ, Tabios M, Vysotskaia V, Zhao L, Andrade RS, et al (2004) Systematic generation of high-resolution deletion coverage of the Drosophila melanogaster genome. Nat Genet 36:288-292.

Pielage J, Fetter RD, Davis GW (2005) Presynaptic spectrin is essential for synapse stabilization. Curr Biol 15:918-928.

Pilling AD, Horiuchi D, Lively CM, Saxton WM (2006) Kinesin-1 and Dynein are the primary motors for fast transport of mitochondria in Drosophila motor axons. Mol Biol Cell 17:2057-2068.

Pulipparacharuvil S, Akbar MA, Ray S, Sevrioukov EA, Haberman AS, Rohrer J, Krämer H (2005) Drosophila Vps16A is required for trafficking to lysosomes and biogenesis of pigment granules. J Cell Sci 118:3663-3673.

Quatacker J, Annaert W, de Potter W (1995) The organisation of the axonal reticulum at a ligation, in in vitro incubated bovine splenic nerves. Brain Res 680:36-42.

Rao SS, Stewart BA, Rivlin PK, Vilinsky I, Watson BO, Lang C, Boulianne G, Salpeter MM, Deitcher DL (2001) Two distinct effects on neurotransmission in a temperature-sensitive SNAP-25 mutant. EMBO J 20:6761-6771.

Roos J, Hummel T, Ng N, Klämbt C, Davis GW (2000) Drosophila Futsch regulates synaptic microtubule organization and is necessary for synaptic growth. Neuron 26:371-382.

Satoh D, Sato D, Tsuyama T, Saito M, Ohkura H, Rolls MM, Ishikawa F, Uemura T (2008) Spatial control of branching within dendritic arbors by dynein-dependent transport of Rab5-endosomes. Nat Cell Biol 10:1164-1171.

Shen W, Ganetzky B (2009) Autophagy promotes synapse development in Drosophila. J Cell Biol 187:71-79.

Sun Y, Aiga M, Yoshida E, Humbert PO, Bamji SX (2009) Scribble interacts with beta-catenin to localize synaptic vesicles to synapses. Mol Biol Cell 20:3390-3400.

Sweeney ST, Davis GW (2002) Unrestricted synaptic growth in spinster-a late endosomal protein implicated in TGF-beta-mediated synaptic growth regulation. Neuron 36:403-416.

Thibault ST, Singer MA, Miyazaki WY, Milash B, Dompe NA, Singh CM, Buchholz R, Demsky M, Fawcett R, Francis-Lang HL, Ryner L, Cheung LM, Chong A, Erickson C, Fisher WW, Greer K, Hartouni SR, Howie E, Jakkula L, Joo D, et al (2004) A complementary transposon tool kit for Drosophila melanogaster using P and piggyBac. Nat Genet 36:283-287.

Toda H, Mochizuki H, Flores R 3rd, Josowitz R, Krasieva TB, Lamorte VJ, Suzuki E, Gindhart JG, Furukubo-Tokunaga K, Tomoda T (2008) UNC-51/ATG1 kinase regulates axonal transport by mediating motorcargo assembly. Genes Dev 22:3292-3307.

Vaillend C, Poirier R, Laroche S (2008) Genes, plasticity and mental retardation. Behav Brain Res 192:88-105.

Wang D, Zhang L, Zhao G, Wahlström G, Heino TI, Chen J, Zhang YQ (2010) Drosophila twinfilin is required for cell migration and synaptic endocytosis. J Cell Sci 123:1546-1556.

Weible MW 2nd, Hendry IA (2004) What is the importance of multivesicular bodies in retrograde axonal transport in vivo? J Neurobiol 58:230-243.

Welte MA (2004) Bidirectional transport along microtubules. Curr Biol 14:R525-R537.

Yao A, Jin S, Li X, Liu Z, Ma X, Tang J, Zhang YQ (2011) Drosophila FMRP regulates microtubule network formation and axonal transport of mitochondria. Hum Mol Genet 20:51-63.

Yu SY, Yoo SJ, Yang L, Zapata C, Srinivasan A, Hay BA, Baker NE (2002) A pathway of signals regulating effector and initiator caspases in the developing Drosophila eye. Development 129:3269-3278.

Yue Z (2007) Regulation of neuronal autophagy in axon: implication of autophagy in axonal function and dysfunction/degeneration. Autophagy 3:139-141.

Zhai RG, Vardinon-Friedman H, Cases-Langhoff C, Becker B, Gundelfinger ED, Ziv NE, Garner CC (2001) Assembling the presynaptic active zone: a characterization of an active one precursor vesicle. Neuron 29:131-143.

Zhang YQ, Rodesch CK, Broadie K (2002) Living synaptic vesicle marker: synaptotagmin-GFP. Genesis 34:142-145.

Zhang Y, Chen D, Wang Z (2009) Analyses of mental dysfunction-related ACS14 in Drosophila reveal its requirement for Dpp/BMP production and visual wiring in the brain. Hum Mol Genet 18:3894-3905. 